FedUni ResearchOnline

https://researchonline.federation.edu.au

This is the author's accepted version of the following publication:

Fabian, M., Henrion, R., Kruger, A., Outrata, J. (2010) Error

bounds: Necessary and sufficient conditions. Set-Valued and

Variational Analysis, 18(2), 121-149.

The version displayed here may differ from the final published version.

The final publication is available at:

http://dx.doi.org/10.1007/s11228-010-0133-0

Copyright $@ 2010$, Springer. 


\title{
Error bounds: necessary and sufficient conditions
}

\author{
Marian J. Fabian • René Henrion . \\ Alexander Y. Kruger • Jiří V. Outrata
}

Received: date / Accepted: date

\begin{abstract}
The paper presents a general classification scheme of necessary and sufficient criteria for the error bound property incorporating the existing conditions. Several derivative-like objects both from the primal as well as from the dual space are used to characterize the error bound property of extended-real-valued functions on a Banach space.
\end{abstract}

Keywords Error bounds · Calmness · Subdifferential · Slope

Mathematics Subject Classification (2000) 49J52 - 49J53 · 58C06

\section{Introduction}

Since the fundamental works of Hoffman [21] and Lojasiewicz [36], the notion of (local) error bound plays a key role in variational analysis. Having a closed set $A$ and a function $f$ with the property that $A=\{x \mid f(x) \leq 0\}$, the principal question reads as follows: For a given $\bar{x} \in A$, does there exist a neighborhood $\mathcal{U}$ of $\bar{x}$ and positive constants $c, \beta$ such that

The research of Marian J. Fabian was supported by Institutional Research Plan of the Academy of Sciences of Czech Republic AVOZ 101905 03, and by GAČR 201/07/0394

The research of René Henrion was supported by the DFG Research Center MATHEON "Mathematics for key technologies" in Berlin

The main structure of the article was developed during Alexander Y. Kruger's stay at the Institute of Information Theory and Automation of the Academy of Sciences of the Czech Republic in July-August 2008; this author is grateful to the Institute for support and hospitality

The research of Jiří V. Outrata was supported by IAA 100750802 of the Grant Agency of the Czech Academy of Sciences

M. Fabian

Mathematical Institute, Academy of Sciences of the Czech Republic, Žitná 25, 11567 Prague 1, Czech Republic

E-mail: fabian@math.cas.cz

R. Henrion

Weierstrass Institute for Applied Analysis and Stochastics, 10117 Berlin, Germany

E-mail: henrion@wias-berlin.de

\section{A. Kruger}

School of Information Technology and Mathematical Sciences, Centre for Informatics and Applied Optimization, University of Ballarat, POB 663, Ballarat, Vic, 3350, Australia

Tel.: +61-3-53279804

Fax: +61-3-53279289

E-mail: a.kruger@ballarat.edu.au

J. Outrata

Institute of Information Theory and Automation, Academy of Sciences of the Czech Republic, 18208 Prague, Czech Republic

E-mail: outrata@utia.cas.cz 


$$
d(x, A) \leq c\left([f(x)]_{+}\right)^{\beta} \text { for all } x \in \mathcal{U} ?
$$

Particularly significant is the case $\beta=1$, when we say that $f$ has a (local) error bound of $A$ of order 1 at $\bar{x}$ or, simply, that $f$ satisfies the error bound property at $\bar{x}$. Property (1) then turns out to be of great importance not only in consistence or optimization problems, but has a deep relationship to subdifferential calculus, optimality conditions, stability and sensitivity issues, convergence of numerical methods, etc. Let us mention several notions of variational analysis closely related to the error bound property.

Metric subregularity was introduced by Ioffe in [22] (under a different name) as a constraint qualification related to equality constraints in nonsmooth mathematical programs. Later it was generalized in [16] to constraints of the form

$$
y \in F(x),
$$

where $F$ is a multifunction and $y$ is fixed. We say that $F$ is metrically subregular at a point $(\bar{x}, \bar{y})$ from the graph of $F$, provided there is a neighborhood $\mathcal{U}$ of $\bar{x}$ and a positive constant $c$ such that

$$
d\left(x, F^{-1}(\bar{y})\right) \leq c d(\bar{y}, F(x)) \text { for all } x \in \mathcal{U} .
$$

This means that $d(\bar{y}, F(\cdot))$ satisfies the error bound property at $\bar{x}$.

Ye and Ye introduced in [52] another important property of multifunctions for which later the term calmness was coined in [48]. A multifunction $M: Y \Rightarrow X$ is called calm at a point $(\bar{y}, \bar{x})$ from its graph, provided there is a positive constant $k$ such that for any $(y, x)$ sufficiently close to $(\bar{y}, \bar{x})$ and such that $x \in M(y)$ one has

$$
d(x, M(\bar{y})) \leq k d(y, \bar{y}) .
$$

It can easily be verified that $F$ is metrically subregular at $(\bar{x}, \bar{y})$ if and only if $M=F^{-1}$ is calm at $(\bar{y}, \bar{x})$ with the same constant. Both these properties play a central role in subdifferential calculus and so, a fortiori, in optimality conditions and various stability issues.

The notions of subregularity and calmness are closely related to the so-called calmness of Clarke [10] and another calmness notion defined in [6]. Both latter properties concern mathematical programs with perturbed constraint sets and depend thus also on the respective objectives. As observed in [20], however, in the case of a Lipschitz objective, the Clarke's calmness boils down to the calmness of the canonically perturbed constraint set in the sense of inequality (3). So, in such a case the relationship to the error bound property can easily be established.

The concept of weak sharp minima [7-9,46,49], very important in numerics, can be considered as another interpretation of the error bound property. In the context of an optimization problem

minimize $f(x)$ subject to $x \in C$,

calmness of the multifunction $y \mapsto\{x \in C \mid f(x) \leq y\}$ at local solutions (or equivalently, metric subregularity of the inverse multifunction) amounts to these solutions being weak sharp local minimizers (see, e.g., [7,49]).

A huge literature deals with the error bound property either of general functions or of functions related to sets of particular structure. These sets range from standard feasible sets in mathematical programming given by equalities and inequalities up to general structures of the form $A:=C \cap$ $F^{-1}(y)$, where $C$ is a closed set and $F$ is a multifunction. We refer the interested reader to the surveys by Azé [1], Lewis and Pang [35], and Pang [44].

Numerous attempts have been made to provide characterizations and criteria for the error bound property in terms of various derivative-like objects which live either in the primal space (directional derivatives, slopes, etc.) or in the dual space (subdifferentials, normal cones) $[4,5,12,15,18-20$, $23,25,39,40,42-45,49-51]$. As to our knowledge, one of the first papers of this kind was [23] in which sufficient conditions for the metric subregularity of a constraint system were stated in terms of the Clarke subdifferential. Its main idea has been used several times in various contexts (e.g. $[25,45,49]$ ) with various subdifferentials (Fréchet, limiting, outer). A different subdifferential criterion was obtained in $[18,19]$ as a by-product in the investigation of calmness of a standard constraint system in the sense of [48]. Another criterion was worked out in [20] on the basis of a primal-type estimate; further important results of this nature can be found in $[13,42,43,45]$. 
This paper goes in the same direction by employing several groups of derivative-like objects both from the primal as well as from the dual space. Our conditions concern mainly the case when $f$ is an extended-real-valued lower semicontinuous function defined on a Banach space $X$ although the majority of the primal space estimates are valid in (not necessarily complete) metric spaces. We provide also specialized results tailored to less general situations. Another aim is to present a general classification scheme of necessary and sufficient conditions for the error bound property incorporating the existing conditions, cf. Fig. $3-6$.

Naturally, throughout the whole study we make extensive use of the notions and tools of modern variational analysis, cf. [38,48]. We also introduce some new derivative-like objects including "uniform" subdifferentials and slopes, convenient for providing natural characterizations of the error bound property.

The plan of the paper is as follows. In Section 2 we establish lower and upper estimates for the error bound modulus in terms of uniform strict slopes. Some other primal space slopes are introduced and investigated in Section 3. They are used for formulating several sufficient criteria for the verification of the error bound property in general Banach spaces. Section 4 is devoted to dual criteria in terms of subdifferential slopes. The main results are formulated in the Asplund space setting. In Sections 5 and 6 we consider finite dimensional and convex cases respectively.

Our basic notation is standard. The closed unit balls in the normed space $X$ and its dual are denoted $\mathbb{B}$ and $\mathbb{B}^{*}$ respectively. $B_{\rho}(x)=x+\rho \mathbb{B}$ denotes the closed ball with radius $\rho$ and center $x$. $d(x, A)=\inf _{a \in A}\|x-a\|$ is the point-to-set distance. The lower $\alpha$-level set $\{x \in X: f(x) \leq \alpha\}$ of an extended-real-valued function $f$ is denoted $[f \leq \alpha]$. We also use the denotation $\alpha_{+}=\max (\alpha, 0)$.

\section{Error Bounds: Necessary and Sufficient Conditions}

In this paper $f$ is an extended-real-valued function on a normed linear space $X,|f(\bar{x})|<\infty$. We are looking for characterizations of the error bound property.

Definition $1 f$ satisfies the error bound property at $\bar{x}$ if there exists a $c>0$ such that

$$
d(x,[f \leq f(\bar{x})]) \leq c(f(x)-f(\bar{x}))_{+} \quad \text { for all } x \text { near } \bar{x} .
$$

Obviously this property can be equivalently defined in terms of the error bound modulus (also known as conditioning rate $[45])$ :

$$
\operatorname{Er} f(\bar{x}):=\liminf _{\substack{x \rightarrow \bar{x} \\ f(x)>f(\bar{x})}} \frac{f(x)-f(\bar{x})}{d(x,[f \leq f(\bar{x})])},
$$

namely, the error bound property holds for $f$ at $\bar{x}$ if and only if $\operatorname{Er} f(\bar{x})>0$.

To formulate criteria for the error bound property we are going to use the following (possibly infinite) nonnegative constants

$$
\begin{aligned}
& -\overline{\mid \nabla f}^{\diamond}(\bar{x}):=\liminf _{x \rightarrow \bar{x}, f(x) \downarrow f(\bar{x})} \sup _{0<\|u-x\|<d(x,[f \leq f(\bar{x})])} \frac{(f(x)-f(u))_{+}}{\|u-x\|}, \\
& +\overline{|\nabla f|^{\diamond}}(\bar{x}):=\liminf _{\substack{x \rightarrow \bar{x}, f(x) \downarrow f(\bar{x}) \\
\alpha-d(x,[f \leq f(\bar{x})]) \downarrow 0}} \sup _{0<\|u-x\|<\alpha} \frac{(f(x)-f(u))_{+}}{\|u-x\|}, \\
& \circ \overline{|\nabla f|^{\diamond}}(\bar{x}):=\liminf _{x \rightarrow \bar{x}, f(x) \downarrow f(\bar{x})} \sup _{u \neq x, f(u) \geq f(\bar{x})} \frac{(f(x)-f(u))_{+}}{\|u-x\|},
\end{aligned}
$$

which, each in its own way, characterize quantitatively the descent rate of the function $f$ near $\bar{x}$. Note that only points $x \notin[f \leq f(\bar{x})]$, that is with $f(x)>f(\bar{x})$, are taken into account when computing (6) - (8). These constants provide uniform (for all $x \notin[f \leq f(\bar{x})]$ in a neighborhood of $\bar{x})$ lower estimates of the corresponding descent rates.

By analogy with the (strong) slope [14] (see also [2,24])

$$
|\nabla f|(\bar{x})=\limsup _{x \rightarrow \bar{x}} \frac{(f(\bar{x})-f(x))_{+}}{\|x-\bar{x}\|},
$$


we are going to call constants (6) - (8) the lower, upper and middle uniform strict slopes of $f$ at $\bar{x}$ respectively. The term "strict" in the names of these (and some subsequent) constants reflects the fact that (6) - (8), being in a sense derivative-like objects, accumulate information about "differential" properties of the function in a neighborhood of the given point and thus can be considered analogs of the strict derivative. The relationships between the constants are given by the next theorem.

Theorem 1 (i). $\quad-\overline{|\nabla f|^{\diamond}}(\bar{x}) \leq{ }^{\circ} \overline{|\nabla f|^{\diamond}}(\bar{x}) \leq+\overline{|\nabla f|^{\diamond}}(\bar{x})$.

(ii). If $f$ is Lipschitz continuous near $\bar{x}$ then $-\overline{|\nabla f|^{\diamond}}(\bar{x})={ }^{\circ} \overline{|\nabla f|^{\diamond}}(\bar{x})=+\left.{ }^{+\nabla f}\right|^{\diamond}(\bar{x})$.

Proof (i) The first inequality follows from definitions (6) and (8): it is sufficient to notice that inequality $\|u-x\|<d(x,[f \leq f(\bar{x})])$ implies $f(u)>f(\bar{x})$. We only need to prove the second inequality. Let $f(x)>f(\bar{x}), \alpha>d(x,[f \leq f(\bar{x})]), f(u) \geq f(\bar{x})$, and $u \neq x$. If $\|u-x\|<d(x,[f \leq$ $f(\bar{x})])$ then

$$
\frac{(f(x)-f(u))_{+}}{\|u-x\|} \leq \sup _{0<\|w-x\|<\alpha} \frac{(f(x)-f(w))_{+}}{\|w-x\|}
$$

Let $\|u-x\| \geq d(x,[f \leq f(\bar{x})])$. For any $\beta \in(d(x,[f \leq f(\bar{x})]), \alpha)$ there is a $w \in[f \leq f(\bar{x})]$ such that $\|w-x\| \leq \beta$. It follows that $f(x)>f(w), f(u) \geq f(w)$, and

$$
\frac{(f(x)-f(u))_{+}}{\|u-x\|} \leq \frac{f(x)-f(w)}{d(x,[f \leq f(\bar{x})])} \leq \frac{\beta}{d(x,[f \leq f(\bar{x})])} \sup _{0<\|w-x\|<\alpha} \frac{f(x)-f(w)}{\|w-x\|} .
$$

Passing to the limit as $\beta \downarrow d(x,[f \leq f(\bar{x})])$ in the last inequality, we arrive at the same estimate (10). The conclusion follows from definitions (7) and (8).

(ii) Thanks to (i) we need only prove the inequality ${ }^{+} \overline{|\nabla f|^{\diamond}}(\bar{x}) \leq-\overline{|\nabla f|^{\diamond}}(\bar{x})$. If $+\overline{|\nabla f|^{\diamond}}(\bar{x})=0$

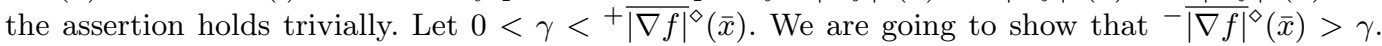
Chose a $\gamma^{\prime} \in\left(\gamma,{ }^{+} \overline{|\nabla f|}^{\diamond}(\bar{x})\right)$. By definition (7), there exists a $\delta>0$ such that for any $x \in X$ satisfying $\|x-\bar{x}\|<\delta$ and $0<f(x)-f(\bar{x})<\delta$ there exists a sequence $\left\{x_{k}\right\} \subset X$ such that $0<\left\|x_{k}-x\right\|<d(x,[f \leq f(\bar{x})])+1 / k$ and $f(x)-f\left(x_{k}\right)>\gamma^{\prime}\left\|x_{k}-x\right\|, k=1,2, \ldots$ If $\left\|x_{k}-x\right\|<$ $d(x,[f \leq f(\bar{x})])$ for some $k$ then

$$
\sup _{0<\|u-x\|<d(x,[f \leq f(\bar{x})])} \frac{(f(x)-f(u))_{+}}{\|u-x\|}>\gamma,
$$

and, by definition $(6),-\left.\overline{|\nabla f|}\right|^{\diamond}(\bar{x})>\gamma$. Suppose that $\left\|x_{k}-x\right\| \geq d(x,[f \leq f(\bar{x})]), k=1,2, \ldots$

Without loss of generality we can assume that $f$ is Lipschitz continuous on $B_{2 \delta}(\bar{x})$ with modulus $l$. For any $k$, consider a point

$$
\hat{x}_{k}=x_{k}+\frac{x-x_{k}}{k\left\|x_{k}-x\right\|} .
$$

It holds

$$
\left\|\hat{x}_{k}-x\right\|=\left|\left\|x_{k}-x\right\|-1 / k\right|, \quad\left\|\hat{x}_{k}-x_{k}\right\|=1 / k .
$$

Let $k>d(x,[f \leq f(\bar{x})])^{-1}$. Then $\left\|x_{k}-x\right\|>1 / k$, and it follows from the first of the above equalities that

$$
\left\|\hat{x}_{k}-x\right\|=\left\|x_{k}-x\right\|-1 / k<d(x,[f \leq f(\bar{x})]) .
$$

At the same time, for large $k$ we have $x_{k}, \hat{x}_{k} \in B_{2 \delta}(\bar{x})$, and consequently,

$$
\frac{f(x)-f\left(\hat{x}_{k}\right)}{\left\|\hat{x}_{k}-x\right\|} \geq \frac{f(x)-f\left(x_{k}\right)-l\left\|\hat{x}_{k}-x_{k}\right\|}{\left\|\hat{x}_{k}-x\right\|}>\frac{\gamma^{\prime}\left\|x_{k}-x\right\|-l / k}{\left\|x_{k}-x\right\|-1 / k}=\gamma^{\prime}-\frac{l-\gamma^{\prime}}{k\left\|x_{k}-x\right\|-1}>\gamma
$$

if $k$ is large enough. Hence, (11) holds true and consequently, ${ }^{-} \overline{|\nabla f|^{\diamond}}(\bar{x})>\gamma$.

The inequalities in Theorem 1 (i) can be strict. 
Example 1 Let $f: \mathbb{R} \rightarrow \mathbb{R}$ be defined as follows:

$$
f(x)= \begin{cases}-1, & \text { if } x<0 \\ x, & \text { if } x \geq 0\end{cases}
$$

Clearly, ${ }^{-} \overline{|\nabla f|^{\diamond}}(0)={ }^{\circ} \overline{|\nabla f|^{\diamond}}(0)=1$ while $+\overline{|\nabla f|^{\diamond}}(0)=\infty$.

The function in the above example is discontinuous at 0 and the upper slope is infinite. In the next example the function is continuous at 0 , the upper slope is finite, and nevertheless it still differs from the lower one.

Example 2 Let $f: \mathbb{R}^{2} \rightarrow \mathbb{R}$ be defined as follows:

$$
f\left(x_{1}, x_{2}\right)= \begin{cases}\min \left(x_{1}, x_{2}\right), & \text { if } x_{1} \geq 0, x_{2} \geq 0 \\ -x_{1}, & \text { if } x_{1}>0, x_{2}<0 \\ -x_{2}, & \text { if } x_{1}<0, x_{2}>0 \\ \max \left(x_{1}, x_{2}\right), & \text { if } x_{1} \leq 0, x_{2} \leq 0\end{cases}
$$

and let $\mathbb{R}^{2}$ be equipped with the Euclidean norm. The function is discontinuous on the set $\{(t, 0) \in$ $\left.\mathbb{R}^{2}: t>0\right\} \cup\left\{(0, t) \in \mathbb{R}^{2}: t>0\right\}$. Then $\left.{ }^{-} \overline{\mid \nabla f}\right|^{\diamond}(0)={ }^{\circ} \overline{|\nabla f|^{\diamond}}(0)=1$ while $^{+} \overline{|\nabla f|^{\diamond}}(0)=2$.

Indeed, let $x=\left(x_{1}, x_{2}\right)$ with $x_{1} \geq x_{2}>0$. Then $f(x)=x_{2}$ and $d(x,[f \leq 0])=x_{2}$. Obviously,

$$
\begin{gathered}
\sup _{0<\|u-x\|<d(x,[f \leq 0])} \frac{(f(x)-f(u))_{+}}{\|u-x\|}=\sup _{\substack{u \neq x, f(u) \geq 0 \\
\lim _{\alpha \downarrow d(x,[f \leq 0])} \sup _{0<\|u-x\|<\alpha}}} \frac{(f(x)-f(u))_{+}}{\|u-x\|}=\frac{x_{1}+x_{2}}{x_{2}} .
\end{gathered}
$$

The last expression attains its minimum value 2 when $x_{1}=x_{2}$. The definition of the function is symmetrical and, consequently, the same conclusion is valid for the case $x_{2} \geq x_{1}>0$. It follows that $-\overline{|\nabla f|}^{\diamond}(0)={ }^{\circ} \overline{|\nabla f|^{\diamond}}(0)=1,{ }^{+} \overline{|\nabla f|^{\diamond}}(0)=2$.

In the next example, the lower slope differs from the middle one.

Example 3 Let $f: \mathbb{R}^{2} \rightarrow \mathbb{R}$ be defined as follows:

$$
f\left(x_{1}, x_{2}\right)= \begin{cases}x_{1}+x_{2}, & \text { if } x_{1}>0, x_{2}>0 \\ 0, & \text { otherwise }\end{cases}
$$

and let $\mathbb{R}^{2}$ be equipped with the Euclidean norm. The function is discontinuous on the set $\{(t, 0) \in$ $\left.\mathbb{R}^{2}: t>0\right\} \cup\left\{(0, t) \in \mathbb{R}^{2}: t>0\right\}$. Then ${ }^{-} \overline{|\nabla f|^{\diamond}}(0)=\sqrt{2}$ while ${ }^{\circ} \overline{|\nabla f|^{\diamond}}(0)={ }^{+}|\overline{\mid \nabla f}|^{\diamond}(0)=2$.

Indeed, let $x=\left(x_{1}, x_{2}\right)$ with $x_{1}>0$ and $x_{2}>0$. Then $d(x,[f \leq 0])=\min \left(x_{1}, x_{2}\right)$. Obviously,

$$
\begin{gathered}
\sup _{0<\|u-x\|<d(x,[f \leq 0])} \frac{(f(x)-f(u))_{+}}{\|u-x\|}=\sup _{\left\|\left(v_{1}, v_{2}\right)\right\|=1}\left(v_{1}+v_{2}\right)=\sqrt{2}, \\
\sup _{u \neq x, f(u) \geq 0} \frac{(f(x)-f(u))_{+}}{\|u-x\|}=\lim _{\alpha \downarrow d(x,[f \leq 0]) \quad 0<\|u-x\|<\alpha} \frac{\sup _{0<\|<} \frac{(f(x)-f(u))_{+}}{\|u-x\|}=\frac{x_{1}+x_{2}}{\min \left(x_{1}, x_{2}\right)} .}{}
\end{gathered}
$$

The last expression attains its minimum value 2 when $x_{1}=x_{2}$. It follows that $-\overline{|\nabla f|^{\diamond}}(0)=\sqrt{2}$, ${ }^{\circ} \overline{\nabla f \mid}{ }^{\diamond}(0)={ }^{+}|\nabla f|^{\diamond}(0)=2$.

The assertion of Theorem 1 (ii) is true also when either $\operatorname{dim} X<\infty$ and $f$ is continuous near $\bar{x}$ or $f$ is convex (see Proposition 11 and Theorem 5).

The next theorem positions the error bound modulus (5) in between upper and middle uniform strict slopes (7) and (8), providing double-sided estimates for the error bound modulus and hence necessary and sufficient criteria for the error bound property.

Theorem 2 (i). $\operatorname{Er} f(\bar{x}) \leq+\overline{\mid}^{\nabla f \mid}{ }^{\diamond}(\bar{x})$.

(ii). If $X$ is Banach and $f$ is lower semicontinuous near $\bar{x}$ then $\operatorname{Er} f(\bar{x}) \geq^{\circ} \overline{\left.\nabla f\right|^{\diamond}}(\bar{x})$. 
Proof (i) If $\operatorname{Er} f(\bar{x})=0$ or ${ }^{+}|\overline{\nabla f}|^{\diamond}(\bar{x})=\infty$ the conclusion is trivial. Let $0<\gamma<\operatorname{Er} f(\bar{x})$ and $+\overline{|\nabla f|}^{\diamond}(\bar{x})<\infty$. We are going to show that ${ }^{+} \overline{|\nabla f|}{ }^{\diamond}(\bar{x}) \geq \gamma$. By (5), there is a $\delta>0$ such that

$$
\frac{f(x)-f(\bar{x})}{d(x,[f \leq f(\bar{x})])}>\gamma .
$$

for any $x \in B_{\delta}(\bar{x})$ with $f(x)>f(\bar{x})$. Take any $x \in B_{\delta}(\bar{x})$ with $f(\bar{x})<f(x) \leq f(\bar{x})+\delta$ (The set of such $x$ is nonempty since ${ }^{+}|\overline{\mid \nabla f}|^{\diamond}(\bar{x})<\infty$.) and then any $\beta>d(x,[f \leq f(\bar{x})])$. By (12), one can find a $w \in[f \leq f(\bar{x})] \cap B_{\beta}(x)$ such that

$$
\frac{f(x)-f(w)}{\|x-w\|}>\gamma
$$

It follows that $+\overline{|\nabla f|^{\diamond}}(\bar{x}) \geq \gamma$.

(ii) If $\operatorname{Er} f(\bar{x})=\infty$ the conclusion is trivial. Let $\operatorname{Er} f(\bar{x})<\gamma<\infty$. Then for any $\delta>0$ there is an $x \in B_{\delta \min \left(1 / 2, \gamma^{-1}\right)}(\bar{x})$ such that

$$
0<f(x)-f(\bar{x})<\gamma d(x,[f \leq f(\bar{x})]) .
$$

Without loss of generality we can assume that $f$ is lower semicontinuous on $B_{\delta}(\bar{x})$. Put $\varepsilon=$ $f(x)-f(\bar{x}), g(u)=(f(u)-f(\bar{x}))_{+}$if $u \in B_{\delta}(\bar{x})$ and $g(u)=\infty$ otherwise. Then $g$ is lower semicontinuous and $g(x) \leq \inf g+\varepsilon$. Applying to $g$ the Ekeland variational principle with an arbitrary $\lambda \in\left(\gamma^{-1} \varepsilon, d(x,[f \leq f(\bar{x})])\right.$, one can find a $w$ such that $f(w) \leq f(x),\|w-x\| \leq \lambda$ and

$$
g(u)+(\varepsilon / \lambda)\|u-w\| \geq g(w), \quad \forall u \in X .
$$

Obviously,

$$
\begin{gathered}
\|w-x\|<d(x,[f \leq f(\bar{x})]) \leq\|x-\bar{x}\|, \\
\|w-\bar{x}\| \leq\|w-x\|+\|x-\bar{x}\|<2\|x-\bar{x}\| \leq \delta, \\
f(w) \leq f(x)<f(\bar{x})+\gamma\|x-\bar{x}\| \leq f(\bar{x})+\delta .
\end{gathered}
$$

Besides, $f(w)>f(\bar{x})$ due to the first inequality in (14), and consequently $g(w)=f(w)-f(\bar{x})$. It follows from (13) that

$$
f(u)+(\varepsilon / \lambda)\|u-w\| \geq f(w)
$$

for all $u \in X$ such that $f(u) \geq f(\bar{x})$. Thus,

$$
\sup _{u \neq w, f(u) \geq f(\bar{x})} \frac{f(w)-f(u)}{\|u-w\|} \leq \varepsilon / \lambda<\gamma .
$$

This implies the inequality ${ }^{\circ} \overline{|\nabla f|^{\diamond}}(\bar{x}) \leq \operatorname{Er} f(\bar{x})$.

Remark 1 The proof of Theorem 2 (ii) refines the one of inclusion (c) $\Rightarrow$ (a) in [25, Theorem 2.1].

Without lower semicontinuity the inequality in Theorem 2 (ii) can fail.

Example 4 Let $f: \mathbb{R} \rightarrow \mathbb{R}$ be defined as follows (Fig. 1):

$$
f(x)= \begin{cases}-3 x, & \text { if } x \leq 0, \\ 3 x-\frac{1}{2^{i}}, & \text { if } \frac{1}{2^{i+1}}<x \leq \frac{1}{2^{i}}, i=0,1, \ldots, \\ 2 x, & \text { if } x>1\end{cases}
$$

Obviously, $\operatorname{Er} f(0)=1$ while $\left.{ }^{\circ} \overline{|\nabla f|}\right|^{\diamond}(0)=3$.

Corollary 1 If $X$ is Banach and $f$ is lower semicontinuous near $\bar{x}$ then

$$
\left.{ }^{\circ} \overline{|\nabla f|}\right|^{\diamond}(\bar{x}) \leq \operatorname{Er} f(\bar{x}) \leq+\left.\overline{|\nabla f|}\right|^{\diamond}(\bar{x}) .
$$

If, additionally, $f$ is Lipschitz continuous near $\bar{x}$ then all three constants coincide.

The inequalities in Corollary 1 can be strict. 


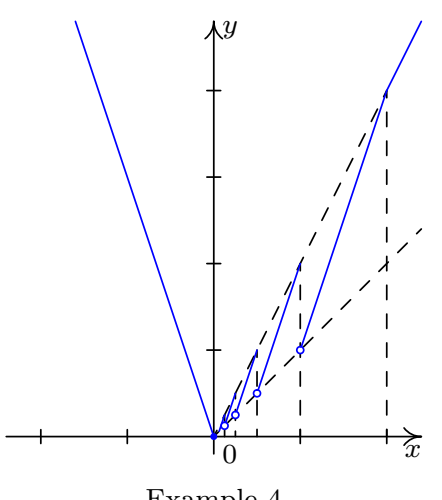

Fig. 1

Example 5 Let $f: \mathbb{R}^{2} \rightarrow \mathbb{R}$ be defined as follows:

$$
f\left(x_{1}, x_{2}\right)= \begin{cases}x_{1}+x_{2}, & \text { if } x_{1}>0, x_{2}>0 \\ -x_{1}, & \text { if } x_{1}>0, x_{2} \leq 0 \\ -x_{2}, & \text { if } x_{2}>0, x_{1} \leq 0 \\ 0, & \text { otherwise }\end{cases}
$$

and let $\mathbb{R}^{2}$ be equipped with the Euclidean norm. The function is discontinuous on the set $\{(t, 0) \in$ $\left.\mathbb{R}^{2}: t>0\right\} \cup\left\{(0, t) \in \mathbb{R}^{2}: t>0\right\}$. Then ${ }^{\circ} \overline{|\nabla f|^{\diamond}}(0)=\sqrt{2}$, Er $f(0)=2$, and ${ }^{+} \overline{|\nabla f|^{\diamond}}(0)=3$.

Indeed, let $x=\left(x_{1}, x_{2}\right)$ with $x_{1} \geq x_{2}>0$. Then $d(x,[f \leq 0])=x_{2}$. Obviously,

$$
\begin{gathered}
\sup _{u \neq x, f(u) \geq 0} \frac{(f(x)-f(u))_{+}}{\|u-x\|}=\sup _{\left\|\left(v_{1}, v_{2}\right)\right\|=1}\left(v_{1}+v_{2}\right)=\sqrt{2}, \\
\frac{f(x)-f(\bar{x})}{d(x,[f \leq f(\bar{x})])}=\frac{x_{1}+x_{2}}{x_{2}}, \\
\lim _{\alpha \downarrow d(x,[f \leq 0])} \sup _{0<\|u-x\|<\alpha} \frac{(f(x)-f(u))_{+}}{\|u-x\|}=\frac{2 x_{1}+x_{2}}{x_{2}} .
\end{gathered}
$$

Expressions (15) and (16) attain their minimum values 2 and 3 respectively when $x_{1}=x_{2}$. The definition of the function is symmetrical and, consequently, the same conclusion is valid for the case $x_{2} \geq x_{1}>0$. It follows that ${ }^{\circ} \overline{|\nabla f|^{\diamond}}(0)=\sqrt{2}$, Er $f(0)=2,+{ }_{|\nabla f|}^{\diamond}(0)=3$.

Due to Theorems 1 and 2, we can formulate the following necessary (NC) and sufficient (C) criteria for the error bound property:

NC1 $+\left.\overline{|\nabla f|}\right|^{\diamond}(\bar{x})>0$.

C1 ${ }^{\circ} \overline{\nabla f \mid}{ }^{\diamond}(\bar{x})>0$.

C2 $-\overline{|\nabla f|}(\bar{x})>0$.

It holds $\mathrm{C} 2 \Rightarrow \mathrm{C} 1 \Rightarrow \mathrm{NC}$. These criteria will be further discussed in the next sections. Note that, in accordance with Theorem 2, conditions $\mathrm{C} 1$ and $\mathrm{C} 2$ as well as the other criteria formulated in the next sections are sufficient under the assumption that $X$ is a Banach space and $f$ is lower semicontinuous near $\bar{x}$.

\section{Primal Space Sufficient Criteria}

This and subsequent sections contain a list of sufficient conditions for the verification of the local error bound property following from Theorem 2 and Corollary 1. Some conditions are new while the others recapture known ones. Wherever possible we provide references to these and similar criteria in the literature. 
For specific functions the choice depends on several circumstances as the type of the underlying space, the structure of the function and the importance of the verification (simple criteria are sometimes far from necessity whereas using finer conditions usually requires a non-negligible effort). We give interrelations among some conditions and provide recommendations about areas of applicability.

For a lower semicontinuous function, conditions $\mathrm{C} 1$ and $\mathrm{C} 2$ provide tight sufficient criteria for the error bound property. In their turn, they are implied by stronger conditions formulated in terms of more conventional primal space derivative-like objects defined on the basis of slopes (9):

$$
\begin{aligned}
& \overline{|\nabla f|}(\bar{x})=\liminf _{(x, f(x)) \rightarrow(\bar{x}, f(\bar{x}))}|\nabla f|(x), \\
& |\overline{|\nabla f|}|^{>}(\bar{x})=\liminf _{x \rightarrow \bar{x}, f(x) \downarrow f(\bar{x})}|\nabla f|(x) .
\end{aligned}
$$

Constants (17) and (18) are called the strict slope and the strict outer slope of $f$ at $\bar{x}$ respectively. In the definition of the last one, the slopes $|\nabla f|(x)$ are computed at points $x$ outside the set $[f \leq f(\bar{x})]$.

Proposition $1 \overline{|\nabla f|}(\bar{x}) \leq \overline{|\nabla f|}^{>}(\bar{x}) \leq-\overline{|\nabla f|}^{\diamond}(\bar{x})$.

Proof The inequalities follow directly from definitions (6), (9), (17), and (18).

The inequalities in Proposition 1 can be strict. The first one can be strict even for convex functions.

Example 6 Let $f: \mathbb{R} \rightarrow \mathbb{R}$ be defined as follows:

$$
f(x)=\left\{\begin{array}{l}
0, \text { if } x<0 \\
x \text { if } x \geq 0 .
\end{array}\right.
$$

The function is convex. Obviously $\overline{|\nabla f|}(0)=|\nabla f|(0)=0$. At the same time, $\mid \overline{\mid \nabla f}^{>}(0)=$ $-\left.\overline{|\nabla f|}\right|^{\diamond}(0)=1$.

See also [45, Example 4.10]. The next example is a modification of the corresponding one in [25]. Example 7 Let $f: \mathbb{R} \rightarrow \mathbb{R}$ be defined as follows (Fig. 2 a):

$$
f(x)= \begin{cases}-x, & \text { if } x \leq 0 \\ \frac{1}{i}, & \text { if } \frac{1}{i+1}<x \leq \frac{1}{i}, i=1,2, \ldots, \\ x, & \text { if } x>1\end{cases}
$$

Obviously $|\nabla f|(x)=0$ for any $x \in(0,1)$, and consequently $\overline{|\nabla f|}>(0)=0$. At the same time, $[f \leq f(0)]=\{0\}, d(x,[f \leq f(0)])=|x|$, and

$$
\sup _{0<|u-x|<|x|} \frac{(f(x)-f(u))_{+}}{|u-x|}=\left\{\begin{array}{l}
\frac{1}{x i}, \text { if } \frac{1}{i+1}<x \leq \frac{1}{i}, i=1,2, \ldots, \\
1, \quad \text { if } x<0 \text { or } x \geq 1 .
\end{array}\right.
$$

It follows from (6) that $-\overline{|\nabla f|}^{\diamond}(0)=1$.

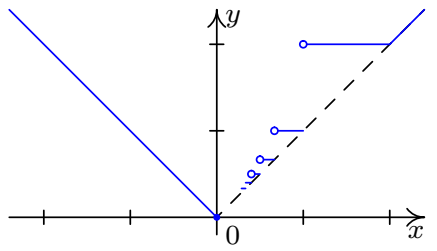

a. Example 7

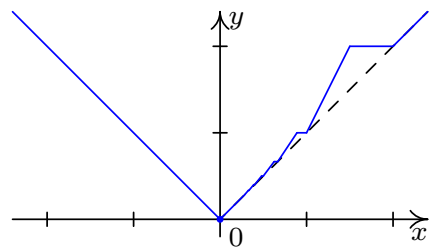

b. Example 8

Fig. 2

The function in the above example is discontinuous. However, the second inequality in Proposition 1 can be strict for continuous and even Lipschitz continuous functions. The function in the next example is piecewise linear and Clarke regular at 0 (that is, directionally differentiable, and its Clarke generalized directional derivative coincides with the usual one). 
Example 8 Let $f: \mathbb{R} \rightarrow \mathbb{R}$ be defined as follows (Fig. 2 b):

$$
f(x)= \begin{cases}-x, & \text { if } x \leq 0, \\ x\left(1+\frac{1}{i}\right)-\frac{1}{i(i+1)}, & \text { if } \frac{1}{i+1}<x \leq \frac{1}{i+1}+\frac{1}{(i+1)^{2}}, i=1,2, \ldots, \\ \frac{1}{i}, & \text { if } \frac{1}{i+1}+\frac{1}{(i+1)^{2}}<x \leq \frac{1}{i}, i=1,2, \ldots, \\ x, & \text { if } x>1 .\end{cases}
$$

The function $f$ is everywhere Fréchet differentiable except for a countable number of points. One can find a point $x>0$ arbitrarily close to 0 with $|\nabla f|(x)=0$ (on a horizontal part of the graph). The slopes of non-horizontal parts of the graph decrease monotonously to 1 as $x \rightarrow 0$. It is not

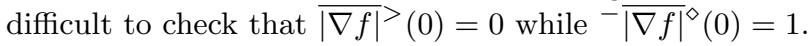

If $f$ is convex then the second inequality in Proposition 1 holds as equality (see Theorem 5).

For the function $f$ in Example 6, it holds $|\nabla f|(0)<\overline{|\nabla f|}^{>}(0)$. In the nonconvex case one can also have the opposite inequality.

Example 9 Let $f: \mathbb{R} \rightarrow \mathbb{R}$ be defined as follows:

$$
f(x)=\left\{\begin{array}{l}
x, \text { if } x<0 \\
x^{2} \text { if } x \geq 0
\end{array}\right.
$$

Obviously $|\nabla f|(0)=1$ while $\mid \overline{|\nabla f|}>(0)=0$. Note that despite the slope $|\nabla f|(0)$ being positive, the function in this example does not satisfy the error bound property at 0 . Hence, condition $|\nabla f|(\bar{x})>0$ is not in general sufficient for the error bound property to hold at $\bar{x}$.

One more constant can be used for providing a lower estimate of the error bound modulus (5):

$$
|\nabla f|^{0}(\bar{x})=\liminf _{x \rightarrow \bar{x}} \frac{f(x)-f(\bar{x})}{\|x-\bar{x}\|} .
$$

Unlike all the other constants considered so far, this one can be negative. In this case it provides a lower estimate of the descent rate of $f$ at $\bar{x}$. More importantly for our purposes, when positive it guarantees that $\bar{x}$ is a point of strict local minimum and provides a lower estimate of the ascent rate of $f$ at $\bar{x}$. Obviously, constant (19) is closely related to (9):

$$
|\nabla f|(\bar{x})=\left(-|\nabla f|^{0}(\bar{x})\right)_{+} .
$$

Proposition 2 (i). $|\nabla f|^{0}(\bar{x}) \leq{ }^{\circ} \overline{|\nabla f|^{\diamond}}(\bar{x})$ (ii). If $|\nabla f|^{0}(\bar{x})>0$ then $|\nabla f|^{0}(\bar{x})=\operatorname{Er} f(\bar{x})$.

Proof If $|\nabla f|^{0}(\bar{x}) \leq 0$ assertion (i) holds trivially. Let $|\nabla f|^{0}(\bar{x})>\gamma>0$. Then by definition (19) there exists a $\delta>0$ such that $f(x)-f(\bar{x})>\gamma\|x-\bar{x}\|$ for all $x \in B_{\delta}(\bar{x}) \backslash\{\bar{x}\}$. Hence,

$$
\inf _{x \in B_{\delta}(\bar{x}) f(x)>f(\bar{x})} \sup _{u \neq x, f(u) \geq f(\bar{x})} \frac{(f(x)-f(u))_{+}}{\|u-x\|}>\gamma,
$$

and consequently ${ }^{\circ} \overline{\left.\nabla f\right|^{\diamond}}(\bar{x}) \geq \gamma$. Hence, ${ }^{\circ} \overline{|\nabla f|^{\diamond}}(\bar{x}) \geq|\nabla f|^{0}(\bar{x})$.

At the same time, $[f \leq f(\bar{x})]=\{\bar{x}\}$ and consequently $d(x,[f \leq f(\bar{x})])=\|x-\bar{x}\|$ for any $x \in X$. The equality in assertion (ii) follows from comparing definitions (5) and (19).

The inequality in Proposition 2 (i) is strict, for instance, when $|\nabla f|^{0}(\bar{x})<0$ (see Examples 1, 2 , and 5). In this case, $|\nabla f|^{0}(\bar{x})$ is obviously smaller than all the other constants. The inequality is also strict for the functions in Examples 3 and 6 where $|\nabla f|^{0}(0)=0$ and for the function in Example 4 where $|\nabla f|^{0}(0)>0$.

For the functions in Examples 3, 6, and 4 one has $\overline{|\nabla f|}^{>}(0)>|\nabla f|^{0}(0)$. On the other hand, for the functions in Examples 7 and 8 it holds $|\nabla f|^{0}(0)>\overline{|\nabla f|}>(0)=\overline{|\nabla f|}(0)=0$. Note also that (20) yields the following implications:

$$
|\nabla f|^{0}(\bar{x})>0 \Rightarrow|\nabla f|(\bar{x})=0, \quad|\nabla f|(\bar{x})>0 \Rightarrow|\nabla f|^{0}(\bar{x})<0 .
$$

Hence, $|\nabla f|(\bar{x})$ and $|\nabla f|^{0}(\bar{x})$ cannot be positive simultaneously.

Due to Propositions 1 and 2 and conditions $\mathrm{C} 1$ and $\mathrm{C} 2$, we can continue the list of sufficient criteria for the error bound property of a lower semicontinuous function on a Banach space: 
C3 $\overline{|\nabla f|}(\bar{x})>0$.

C4 $\overline{|\nabla f|}>(\bar{x})>0$.

C5 $|\nabla f|^{0}(\bar{x})>0$.

An analog of criterion C4 can be found in [42, Corollary 2.3], [45, Theorem 2.10]. Some similar considerations (in terms of multifunctions) can also be found in $[29,30]$.

It holds $\mathrm{C} 3 \Rightarrow \mathrm{C} 4 \Rightarrow \mathrm{C} 2$ and $\mathrm{C} 5 \Rightarrow \mathrm{C} 1$. Criterion $\mathrm{C} 5$ is independent of $\mathrm{C} 4$ and $\mathrm{C} 3$. It can make sense considering the "combined" sufficient criteria

$$
\max \left(\overline{|\nabla f|}(\bar{x}),|\nabla f|^{0}(\bar{x})\right)>0, \quad \max \left(\left.\overline{|\nabla f|}\right|^{>}(\bar{x}),|\nabla f|^{0}(\bar{x})\right)>0 .
$$

The relationships among the primal space error bound criteria for a lower semicontinuous function on a Banach space are illustrated in Fig. 3.

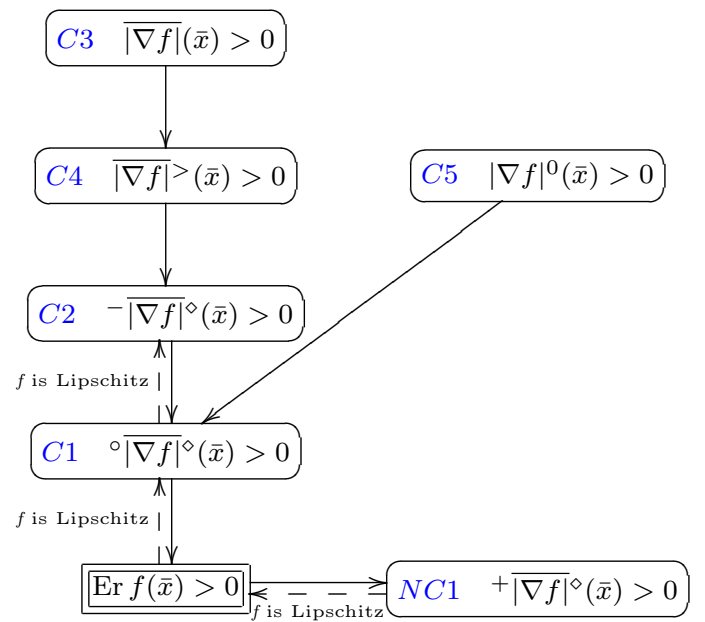

Fig. 3 Primal space criteria

\section{Subdifferential Criteria}

In this section we discuss subdifferential error bounds criteria corresponding to the conditions formulated in the preceding sections in terms of different kinds of (primal space) slopes.

We start with recalling the well known notion of the Fréchet subdifferential of $f$ at $\bar{x}$ (see for instance [34,38]):

$$
\partial f(\bar{x})=\left\{x^{*} \in X^{*}: \liminf _{x \rightarrow \bar{x}} \frac{f(x)-f(\bar{x})-\left\langle x^{*}, x-\bar{x}\right\rangle}{\|x-\bar{x}\|} \geq 0\right\} .
$$

It is a convex subset of $X^{*}$. If $f$ is convex, (21) coincides with the subdifferential in the sense of convex analysis.

Based on (21) one can define the subdifferential slope of $f$ at $\bar{x}$ :

$$
|\partial f|(\bar{x})=\inf \left\{\left\|x^{*}\right\|: x^{*} \in \partial f(\bar{x})\right\} .
$$

It represents the subdifferential counterpart of the slope (9) and can be interpreted as an example of a decrease index [45]. The relationship between the two constants is straightforward.

Proposition $3|\nabla f|(\bar{x}) \leq|\partial f|(\bar{x})$.

Proposition 3 is well known - see [2, Proposition 2.5], [4, Remark 5.3], [24, Proposition 3.2]).

The inequality in Proposition 3 can be strict rather often (for example, if $\partial f(\bar{x})=\emptyset$ ). If $f$ is convex then the two constants coincide (see Theorem 5). 
4.1 Strict subdifferential slopes

The subdifferential counterparts of (17) and (18) can be defined in the following way:

$$
\begin{aligned}
& \overline{|\partial f|}(\bar{x})=\liminf _{(x, f(x)) \rightarrow(\bar{x}, f(\bar{x}))}|\partial f|(x), \\
& \overline{|\partial f|}^{>}(\bar{x})=\liminf _{x \rightarrow \bar{x}, f(x) \downarrow f(\bar{x})}|\partial f|(x) .
\end{aligned}
$$

They are called strict subdifferential slope and strict outer subdifferential slope of $f$ at $\bar{x}$ respectively. Constant (23) was used for characterizing the error bound property in $[2,4,43]$.

Proposition $4 \overline{|\partial f|}(\bar{x}) \leq \overline{|\partial f|}^{>}(\bar{x})$.

The inequality follows directly from definitions (23) and (24). It can be strict even for convex functions, see the function in Example 6.

Proposition 5 (i). $\overline{|\nabla f|}(\bar{x}) \leq \overline{|\partial f|}(\bar{x}) \leq|\partial f|(\bar{x}), \overline{|\nabla f|}^{>}(\bar{x}) \leq \overline{|\partial f|}^{>}(\bar{x})$. (ii). If $X$ is Asplund and $f$ is lower semicontinuous near $\bar{x}$ then $\overline{|\nabla f|}(\bar{x})=\overline{|\partial f|}(\bar{x}), \overline{|\nabla f|}^{>}(\bar{x})=$ $\overline{|\partial f|}>(\bar{x})$.

Proof (i) The inequalities follow from Proposition 3 and definitions (17), (18), (23), and (24).

(ii) Taking into account (i) we only need to prove the opposite inequalities. Let us show that $\overline{|\nabla f|}(\bar{x}) \geq \overline{|\partial f|}(\bar{x})$. If $\overline{|\nabla f|}(\bar{x})=\infty$ the assertion is trivial. Take any $\gamma>\overline{|\nabla f|}(\bar{x})$. We are going to show that $\overline{|\partial f|}(\bar{x}) \leq \gamma$. By definition (17), for any $\beta \in(\mid \overline{|\nabla f|}(\bar{x}), \gamma)$ and any $\delta>0$ there is an $x \in B_{\delta / 2}(\bar{x})$ such that $|f(x)-f(\bar{x})| \leq \delta / 2$ and $|\nabla f|(x)<\beta$. By definition (9), $x$ is a local minimum point of the function $u \mapsto g(u)=f(u)+\beta\|u-x\|$, and consequently $0 \in \partial g(x)$. By the fuzzy (semi-Lipschitzian) sum rule (see for example [38, Theorem 2.33]), this implies the existence of a point $w \in B_{\delta / 2}(x)$ with $|f(w)-f(x)| \leq \delta / 2$ and an element $x^{*} \in \partial f(w)$ with $\left\|x^{*}\right\|<\gamma$. The inequality $\overline{|\partial f|}(\bar{x}) \leq \gamma$ follows from definition (23).

The proof of the other inequality $\overline{|\nabla f|}^{>}(\bar{x}) \geq \overline{|\partial f|}^{>}(\bar{x})$ can be done along the same lines with the obvious replacement of the references to definitions (17) and (23) by those to definitions (18) and (24), and inequality $|f(x)-f(\bar{x})| \leq \delta / 2$ by the following one: $0<f(x)-f(\bar{x}) \leq \delta / 2$.

Remark 2 Equality $\overline{|\nabla f|}(\bar{x})=\overline{|\partial f|}(\bar{x})$ in Proposition 5 (ii) strengthens the inequality

$$
|\nabla f|(\bar{x}) \geq \liminf _{(x, f(x)) \rightarrow(\bar{x}, f(\bar{x}))} d(0, \partial f(x))
$$

in [2, Proposition 2.3], [4, Proposition 4.1].

In the proof of Proposition 5 (ii), three basic properties of Fréchet subdifferentials were used:

1 ) if $x$ is a local minimum point of $f$ then $0 \in \partial f(x)$;

2) if $f$ is convex then $\partial f(x)$ coincides with the subdifferential in the sense of convex analysis;

3) the semi-Lipschitzian sum rule (one function is locally Lipschitz and the other one - lower semicontinuous).

The last property is precisely the place where Asplundity of the space comes into play. Instead of Fréchet subdifferentials, any other subdifferentials possessing the formulated above three properties can be used along the same lines. For example, one can consider Clarke subdifferentials $\partial_{C} f(x)$ $[10,11]$, for which the (exact) semi-Lipschitzian sum rule holds in arbitrary normed spaces [47, Corollary 2.2]. Replacing Fréchet subdifferentials $\partial f(x)$ in the proof of Proposition 5 (ii) by Clarke ones $\partial_{C} f(x)$, we arrive at the following assertion.

Proposition 6 Let $f$ is lower semicontinuous near $\bar{x}$. Then

$$
\begin{aligned}
& \overline{|\nabla f|}(\bar{x}) \geq \liminf _{(x, f(x)) \rightarrow(\bar{x}, f(\bar{x}))} \inf \left\{\left\|x^{*}\right\|: x^{*} \in \partial_{C} f(x)\right\}, \\
& \overline{|\nabla f|}^{>}(\bar{x}) \geq \liminf _{x \rightarrow \bar{x}, f(x) \downarrow f(\bar{x})} \inf \left\{\left\|x^{*}\right\|: x^{*} \in \partial_{C} f(x)\right\} .
\end{aligned}
$$

It is well known (see $[31,38]$ ) that $\partial_{C} f(x)$ is rather often much larger than $\partial f(x)$ (as well as corresponding to it limiting subdifferential), and consequently the inequalities in Proposition 6 are often strict. In Asplund spaces it obviously provides weaker conditions compared to Proposition 5 (ii). 
Remark 3 The first inequality in Proposition 6 is well known - see [2, Proposition 2.3], [4, Proposition 4.1] (where it was formulated for an abstract subdifferential satisfying conditions similar to properties 1) - 3) above).

Thanks to Proposition 5 (ii), the following sufficient subdifferential criteria can be used for characterizing the error bound property of lower semicontinuous functions in Asplund spaces, replacing criteria $\mathrm{C} 4$ and $\mathrm{C} 3$.

C6 $\overline{|\partial f|}(\bar{x})>0$.

C7 $\overline{|\partial f|}{ }^{>}(\bar{x})>0$.

Criterion C7 was used in [43, Corollary 2(ii)], [45, Theorem 4.12], [50, Theorem 3.1]. See also [26, Theorem 2.5].

\subsection{Internal subdifferential slope}

Another subdifferential slope based on the Fréchet subdifferential can be of interest.

A subset $G \subset \partial f(\bar{x})$ is called a regular set of subgradients of $f$ at $\bar{x}$ if for any $\varepsilon>0$ there exists a $\delta>0$ such that

$$
f(x)-f(\bar{x})-\sup _{x^{*} \in G}\left\langle x^{*}, x-\bar{x}\right\rangle+\varepsilon\|x-\bar{x}\| \geq 0, \quad \forall x \in B_{\delta}(\bar{x}) .
$$

The set $\partial f(\bar{x})$ itself does not have to be a regular set of subgradients.

Example 10 Let $f: \mathbb{R} \rightarrow \mathbb{R}$ be defined as follows:

$$
f(x)= \begin{cases}0, & \text { if } x<0 \\ \sqrt{x}, & \text { if } x \geq 0\end{cases}
$$

Obviously $\partial f(0)=[0, \infty)$, and this set is not a regular set of subgradients. Indeed, take $\varepsilon=1$, $x_{k}=1 / k, x_{k}^{*}=k+\sqrt{k}+1, k=1,2, \ldots$ Then $f\left(x_{k}\right)-f(0)-\left\langle x_{k}^{*}, x_{k}\right\rangle+\varepsilon\left|x_{k}\right|=-1$.

Remark 4 The requirement that $\partial f(\bar{x})$ is a regular set of subgradients seems to be a useful regularity property of a real-valued function $f$ at $\bar{x}$. It holds, for instance, for convex functions, see Proposition 15 (i). Compare with a close concept of weak regularity introduced in [27]. Note that the last concept is in general weaker than the one mentioned above, see Example 10 and [27, Example 1].

The set $G$ is defined not uniquely. For instance, any finite subset of $\partial f(\bar{x})$ is a regular set of subgradients of $f$ at $\bar{x}$ and a subset of a regular set of subgradients is also a regular set of subgradients. Another example is given in the next obvious statement.

Proposition 7 For any $\delta>0$ the set of all $x^{*} \in X^{*}$ such that

$$
f(x)-f(\bar{x}) \geq\left\langle x^{*}, x-\bar{x}\right\rangle, \quad \forall x \in B_{\delta}(\bar{x})
$$

is a regular set of subgradients of $f$ at $\bar{x}$.

The next constant, the internal subdifferential slope of $f$ at $\bar{x}$, defined by the equality

$$
|\partial f|^{0}(\bar{x})=\sup \left\{r \geq 0: r \mathbb{B}^{*} \text { is a regular set of subgradients of } f \text { at } \bar{x}\right\},
$$

can be considered as a subdifferential counterpart of the slope defined by (19).

Proposition $8|\partial f|^{0}(\bar{x})=\left(|\nabla f|^{0}(\bar{x})\right)_{+} \cdot{ }^{1}$

\footnotetext{
1 The equality was established by the reviewer.
} 
Proof By definition (25), $|\partial f|^{0}(\bar{x})$ is the exact upper bound of all numbers $r \geq 0$ such that for any $\varepsilon>0$ there exists a $\delta>0$ such that

$$
f(x)-f(\bar{x}) \geq(r-\varepsilon)\|x-\bar{x}\|, \quad \forall x \in B_{\delta}(\bar{x}) .
$$

In other words,

$$
|\partial f|^{0}(\bar{x})=\sup \left\{r \geq 0: r \leq|\nabla f|^{0}(\bar{x})\right\}=\left(|\nabla f|^{0}(\bar{x})\right)_{+} \cdot
$$

Proposition $9|\partial f|^{0}(\bar{x}) \leq \sup \left\{r \geq 0: r \mathbb{B}^{*} \subset \partial f(\bar{x})\right\}$.

This property follows immediately from definition (25). In particular, the inequality $|\partial f|^{0}(\bar{x})>0$ obviously implies the inclusion $0 \in \operatorname{int} \partial f(\bar{x})$.

Thanks to Proposition 8 and condition C5, we can formulate another sufficient subdifferential criteria for the error bound property of lower semicontinuous functions:

C8 $|\partial f|^{0}(\bar{x})>0$.

This criterion is equivalent to C5 and independent of C7 and C6. It can make sense considering the "combined" sufficient criteria

$$
\max \left(\overline{|\partial f|}(\bar{x}),|\partial f|^{0}(\bar{x})\right)>0, \quad \max \left(\overline{|\partial f|}^{>}(\bar{x}),|\partial f|^{0}(\bar{x})\right)>0 .
$$

It follows from the next theorem that in infinite dimensions the inequality in Proposition 9 can be strict.

Theorem 3 In any infinite-dimensional Banach space $(X,\|\cdot\|)$ with a monotone Schauder basis there exists a 1-Lipschitzian function $f: X \rightarrow[0, \infty)$ such that $f(0)=0, f$ is directionally differentiable at 0 with $f^{\prime}(0 ; z)=\|z\|$ for every $z \in X$, and $|\nabla f|^{0}(\bar{x})=\operatorname{Er} f(0)=0$.

Recall [17, Definition 6.1] that a sequence $e_{1}, e_{2}, \ldots$ in a normed linear space $X$ is called a Schauder basis of $X$ if for every $x \in X$ there is a unique sequence $a_{1}, a_{2}, \ldots \in \mathbb{R}$, called coordinates of $x$, such that $\left\|\sum_{i=1}^{n} a_{i} e_{i}-x\right\| \rightarrow 0$ as $n \rightarrow \infty$. For $n \in \mathbb{N}$, the linear mappings $P_{n}: X \rightarrow X$ defined as $P_{n} x=\sum_{i=1}^{n} a_{i} e_{i}$ are called canonical projections. If $X$ is complete then canonical projections are bounded, and one can define an equivalent norm on $X$ by $\|x\| \mid=\sup _{n \in \mathbb{N}}\left\|\sum_{i=1}^{n} a_{i} e_{i}\right\|[17$, Lemma 6.4]. With this norm, the Schauder basis becomes monotone, that is satisfies $\left|\left\|P_{n}\right\|\right|=1$ for every $n \in \mathbb{N}$. It can also be convenient to make the monotone Schauder basis normalized, that is satisfying $\left|\left\|e_{i}\right\|\right|=1$ for every $i \in \mathbb{N}$. In this case, for an $x_{n}^{*} \in X^{*}$ defined by $\left\langle x_{n}^{*}, x\right\rangle=a_{n}$ one has $\left|\left\|x_{n}^{*}\right\|\right|=\left\|\left|P_{n}-P_{n-1} \|\right| \leq 2, n=2,3, \ldots\right.$ Obviously $\|\left|x_{1}^{*} \|\right|=1$.

It should be noted that there are only few known examples of separable Banach spaces without a Schauder basis.

Proof Let $e_{1}, e_{2}, \ldots \in X$ be a normalized monotone Schauder basis in $(X,\|\cdot\|)$. Define

$$
f(x)=\min \left(\|x\|, \inf _{i \in \mathbb{N}}\left\{d\left(x, \mathbb{R} e_{i}\right)+\frac{1}{i^{2}}\right\}\right), \quad x \in X .
$$

Then $f(0)=0$, and $f(x)>0$ if $x \neq 0$. This function is 1 -Lipschitzian as a pointwise infimum of a family of nonnegative 1-Lipschitzian functions. We are going to compute its one-sided directional derivatives at 0 . First take $z$ in the linear span of $\left\{e_{1}, e_{2}, \ldots\right\}$ and denote by $j_{z}$ an index such that $z$ is a linear combination of $\left\{e_{1}, e_{2}, \ldots, e_{j_{z}}\right\}$. If $j>j_{z}$ then the monotonicity of the basis yields that $d\left(z, \mathbb{R} e_{j}\right)=\|z\|$, and consequently

$$
d\left(t z, \mathbb{R} e_{j}\right)+\frac{1}{j^{2}}>t\|z\|
$$

for all $t>0$. If $j \leq j_{z}$ then for sufficiently small $t>0$ we have

$$
d\left(t z, \mathbb{R} e_{j}\right)+\frac{1}{j^{2}} \geq \frac{1}{j_{z}^{2}}>t\|z\| .
$$


It follows from (26) that $f(t z)=t\|z\|$ for all sufficiently small $t>0$. Therefore $f^{\prime}(0 ; z)=\|z\|$. Since the linear span of $\left\{e_{1}, e_{2}, \ldots\right\}$ is dense in $X$ and $f$ is Lipschitzian, we conclude that $f^{\prime}(0 ; z)$ exists for every $z \in X$ and $f^{\prime}(0 ; z)=\|z\|$.

Consider the sequence $x_{i}=e_{i} / i, i \in \mathbb{N}$. Obviously $\left\|x_{i}\right\|=1 / i$. Let $j \in \mathbb{N}$ be different from $i$. We are going to show that $d\left(x_{i}, \mathbb{R} e_{j}\right) \geq 1 /(3 i)$. Indeed, take any $t \in \mathbb{R}$. If $|t|<2 /(3 i)$, then

$$
\left\|x_{i}-t e_{j}\right\| \geq \frac{1}{i}-|t|>\frac{1}{3 i} .
$$

If $|t| \geq 2 /(3 i)$ then

$$
\left\|x_{i}-t e_{j}\right\| \geq \frac{1}{2}\left|\left\langle x_{j}^{*}, \frac{1}{i} e_{i}-t e_{j}\right\rangle\right|=\frac{1}{2}|t| \geq \frac{1}{3 i} .
$$

Having this, we can estimate for $i \geq 3$ :

$$
d\left(x_{i}, \mathbb{R} e_{j}\right)+\frac{1}{j^{2}}>\frac{1}{3 i} \geq \frac{1}{i^{2}}=d\left(x_{i}, \mathbb{R} e_{i}\right)+\frac{1}{i^{2}} .
$$

Hence by (26), $f\left(x_{i}\right)=\min \left(\left\|x_{i}\right\|, 1 / i^{2}\right)=1 / i^{2}$. It follows that $f\left(x_{i}\right) /\left\|x_{i}\right\|=1 / i \rightarrow 0$ as $i \rightarrow \infty$. Hence, $|\nabla f|^{0}(\bar{x})=\operatorname{Er} f(0)=0$.

Corollary 2 In any infinite-dimensional Banach space $X$ with a monotone Schauder basis there exists a function $f: X \rightarrow \mathbb{R}$ such that $\partial_{C} f(0)=\partial f(0)=\mathbb{B}^{*}$ and $|\partial f|^{0}(0)=|\nabla f|^{0}(\bar{x})=0$.

Proof The function the existence of which is guaranteed by Proposition 3 satisfies the conclusions of Corollary 2. Indeed, since $f$ is 1 -Lipschitzian, its Clarke directional derivative at 0 satisfies $f^{\circ}(0 ; z) \leq\|z\|$ for all $z \in X$. Thus, $\|z\|=f^{\prime}(0 ; z) \leq f^{\circ}(0 ; z) \leq\|z\|$. It follows that $f$ is Clarke regular at 0 and $\partial f(0)=\mathbb{B}^{*}$. The conclusion follows from Proposition 8 .

Example 11 Let $f: l^{2} \rightarrow \mathbb{R}$ be defined as follows:

$$
f(x)=\min \left(\|x\|, \inf _{i \in \mathbb{N}}\left\{\left\|P_{i} x\right\|+\frac{1}{i^{2}}\right\}\right),
$$

where $P_{i} x=\left(x_{1}, \ldots, x_{i-1}, 0, x_{i+1}, \ldots\right)$. Then $f(0)=0,[f \leq 0]=\{0\}, \partial f(0)=\mathbb{B}$, and $|\partial f|^{0}(0)=$ $|\nabla f|^{0}(\bar{x})=0$.

\subsection{Uniform strict subdifferential slope}

The following nonlocal modification of the Fréchet subdifferential (21), depending on two parameters $\alpha \geq 0$ and $\varepsilon \geq 0$, can be of interest:

$$
\partial_{\varepsilon, \alpha}^{\diamond} f(\bar{x})=\left\{x^{*} \in X^{*}: \sup _{\beta>\alpha} \inf _{0<\|x-\bar{x}\| \leq \beta} \frac{f(x)-f(\bar{x})-\left\langle x^{*}, x-\bar{x}\right\rangle}{\|x-\bar{x}\|} \geq-\varepsilon\right\} .
$$

We are going to call $(27)$ the uniform $(\varepsilon, \alpha)$-subdifferential of $f$ at $\bar{x}$. Obviously it is a convex set in $X^{*}$. When $\alpha=0$ it coincides with the $\varepsilon$-subdifferential [32-34] of $f$ at $\bar{x}$, and

$$
\partial f(\bar{x})=\bigcap_{\varepsilon>0} \partial_{\varepsilon, 0}^{\diamond} f(\bar{x}) .
$$

Using uniform $(\varepsilon, \alpha)$-subdifferentials (27) one can define the uniform strict subdifferential slope of $f$ at $\bar{x}$ - a subdifferential counterpart of the upper uniform strict slope (7):

$$
\overline{|\partial f|^{\diamond}}(\bar{x})=\liminf _{x \rightarrow \bar{x}, f(x) \downarrow f(\bar{x}), \varepsilon \downarrow 0} \inf \left\{\left\|x^{*}\right\|: x^{*} \in \partial_{\varepsilon, d(x,[f \leq f(\bar{x})])}^{\diamond} f(x)\right\} .
$$

Note that in the definition (28) of the uniform strict subdifferential slope, the uniform $(\varepsilon, \alpha)$ subdifferentials are taken with $\alpha$ depending on $x$, namely $\alpha=d(x,[f \leq f(\bar{x})])$. This number is the same for all points $x$ with the same distance from the set $[f \leq f(\bar{x})]$ and goes to zero as $x$ approaches $\bar{x}$.

Proposition $10(i) .{ }^{+} \overline{|\nabla f|}^{\diamond}(\bar{x}) \leq \overline{\mid \partial f}^{\diamond}(\bar{x})$. 
(ii). Suppose that the following uniformity condition holds true for $f$ :

(UC) There is $a \delta>0$ and a function $o: \mathbb{R}_{+} \rightarrow \mathbb{R}$ such that $\lim _{t \downarrow 0} o(t) / t=0$ and for any $x \in B_{\delta}(\bar{x})$ with $0<f(x)-f(\bar{x}) \leq \delta$ and any $x^{*} \in \partial f(x)$ it holds

$$
f(u)-f(x)-\left\langle x^{*}, u-x\right\rangle+o(\|u-x\|) \geq 0, \quad \forall u \in X .
$$

Then $\overline{|\partial f|^{\diamond}}(\bar{x}) \leq \overline{|\partial f|}^{>}(\bar{x})$.

(iii). If $X$ is Banach and $f$ is lower semicontinuous near $\bar{x}$ and satisfies the uniformity condition (UC) then

$$
\operatorname{Er} f(\bar{x})=\overline{|\partial f|}^{>}(\bar{x})=\overline{|\nabla f|}^{>}(\bar{x})={ }^{-} \overline{|\nabla f|}{ }^{\diamond}(\bar{x})={ }^{\circ} \overline{|\nabla f|^{\diamond}}(\bar{x})={ }^{+} \overline{|\nabla f|^{\diamond}}(\bar{x})=\overline{\mid \partial f}^{\diamond}(\bar{x}) \geq|\partial f|^{0}(\bar{x}) .
$$

Proof (i) The inequality follows from the definitions.

(ii) If $\overline{|\partial f|}>(\bar{x})=\infty$ the inequality holds true trivially. Let $\overline{|\partial f|}{ }^{>}(\bar{x})<\gamma<\infty$ and $\varepsilon>0$. By definition (24), for any $\delta>0$ there is an $x \in B_{\delta}(\bar{x})$ with $0<f(x)-f(\bar{x}) \leq \delta$ and an $x^{*} \in \partial f(x)$ with $\left\|x^{*}\right\|<\gamma$. Without loss of generality we can take $\delta>0$ small enough such that (29) holds true and $o(t) / t \leq \varepsilon$ if $0<t<2 \delta$. Then

$$
\sup _{\beta>\alpha} \inf _{0<\|u-x\| \leq \beta} \frac{f(u)-f(x)-\left\langle x^{*}, u-x\right\rangle}{\|u-x\|} \geq-\varepsilon,
$$

where $\alpha:=d(x,[f \leq f(\bar{x})]) \leq\|x-\bar{x}\| \leq \delta$. Thus, $x^{*} \in \partial_{\varepsilon, \alpha}^{\diamond} f(x)$, and consequently $\overline{|\partial f|^{\diamond}}(\bar{x})<\gamma$.

(iii) follows from (i) and (ii), Propositions 1, 5 (ii), and 8, Theorem 1 (i) and Corollary 1.

Note that in finite dimensions inequality $|\partial f|^{0}(\bar{x}) \leq \overline{\mid \partial f}^{>}(\bar{x})$ in Proposition 10 (iii) holds true also for semismooth functions (Proposition 14 (iii)).

Taking into account condition NC1, Proposition 10 (i) allows us to formulate another necessary condition for the error bound property.

NC2 $\left.\overline{|\partial f|}\right|^{\diamond}(\bar{x})>0$.

The relationships among the subdifferential error bound criteria for a lower semicontinuous function on an Asplund space are illustrated in Fig. 4.

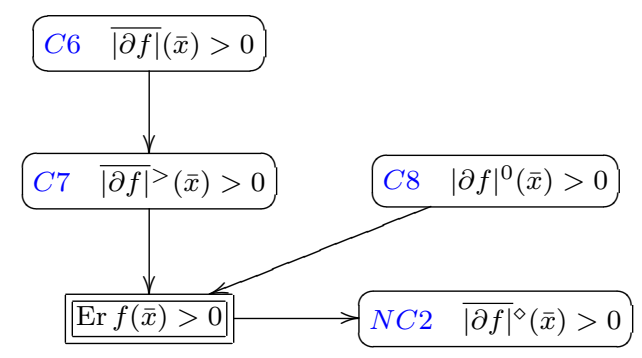

Fig. 4 Subdifferential criteria

Note that sufficient criterion $\mathrm{C} 8$ and necessary criterion $\mathrm{NC} 2$ are applicable in general Banach spaces. Conditions $\mathrm{C} 6$ and $\mathrm{C} 7$ can be replaced by the corresponding criteria in terms of Clarke subdifferentials (Proposition 6) which are also valid in general Banach spaces.

\section{Finite Dimensional Case}

In this section $\operatorname{dim} X<\infty$ and $f$ is lower semicontinuous.

The assertion of Theorem 1 (ii) can be strengthened: there is no need to assume $f$ Lipschitz continuous; in finite dimensions just continuity is sufficient.

Proposition 11 If $f$ is continuous near $\bar{x}$ then ${ }^{-} \overline{|\nabla f|^{\diamond}}(\bar{x})={ }^{\circ} \overline{|\nabla f|^{\diamond}}(\bar{x})={ }^{+} \overline{|\nabla f|^{\diamond}}(\bar{x})=\operatorname{Er} f(\bar{x})$. 
Proof The proof of the first two equalities is similar to that of Theorem 1 (ii). Thanks to Theorem 1 (i) we need only prove the inequality ${ }^{+} \overline{|\nabla f|^{\diamond}}(\bar{x}) \leq-\overline{|\nabla f|^{\diamond}}(\bar{x})$. If $+\frac{+}{|\nabla f|^{\diamond}}(\bar{x})=0$ the assertion holds trivially. Let $0<\gamma<+\overline{|\nabla f|^{\diamond}}(\bar{x})$. We are going to show that ${ }^{-}|\overline{\nabla f}|^{\diamond}(\bar{x})>\gamma$. Chose a $\gamma^{\prime} \in\left(\gamma,{ }^{+} \overline{\nabla f}^{\diamond}(\bar{x})\right)$. By definition (7), there exists a $\delta>0$ such that for any $x \in X$ satisfying $\|x-\bar{x}\|<\delta$ and $0<f(x)-f(\bar{x})<\delta$ there exists a sequence $\left\{x_{k}\right\} \subset X$ such that $0<\left\|x_{k}-x\right\|<d(x,[f \leq f(\bar{x})])+1 / k$ and $f(x)-f\left(x_{k}\right)>\gamma^{\prime}\left\|x_{k}-x\right\|, k=1,2, \ldots$ If

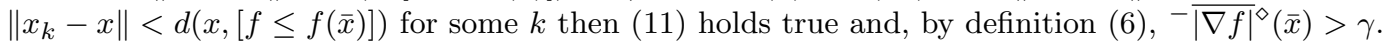
Suppose that $\left\|x_{k}-x\right\| \geq d(x,[f \leq f(\bar{x})]), k=1,2, \ldots$.

Without loss of generality we can assume that $f$ is continuous on $B_{2 \delta}(\bar{x})$. The sequence $\left\{x_{k}\right\}$ has an accumulation point $\hat{x}$ satisfying $\|\hat{x}-x\|=d(x,[f \leq f(\bar{x})]),\|\hat{x}-\bar{x}\|<2 \delta$, and $f(x)-f(\hat{x}) \geq$ $\gamma^{\prime}\|\hat{x}-x\|$. Due to continuity of $f$, it is possible to find a point $u \in X$ sufficiently close to $\hat{x}$ and satisfying $\|u-x\|<d(x,[f \leq f(\bar{x})])$ and $f(x)-f(u)>\gamma\|u-x\|$. Hence, (11) is satisfied, and consequently, $-\left.\overline{|\nabla f|}\right|^{\diamond}(\bar{x})>\gamma$.

The last equality follows from Theorem 2.

The strict subdifferential slopes (23), (24), and (28) can be defined equivalently in terms of the following limiting subdifferentials:

$$
\begin{gathered}
\bar{\partial} f(\bar{x})=\operatorname{Limsup}_{(x, f(x)) \rightarrow(\bar{x}, f(\bar{x}))} \partial f(x), \\
\bar{\partial}^{>} f(\bar{x})=\operatorname{Limsup}_{x \rightarrow \bar{x}, f(x) \downarrow f(\bar{x})} \partial f(x), \\
\bar{\partial}^{\diamond} f(\bar{x})=\operatorname{Limsup}_{x \rightarrow \bar{x}, f(x) \downarrow f(\bar{x}), \varepsilon \downarrow 0} \partial_{\varepsilon, d(x,[f \leq f(\bar{x})])}^{\diamond} f(x) .
\end{gathered}
$$

In the above definitions, Lim sup denotes the outer limit [48] operation for sets: each of the sets (30) - (32) is the set of all limits of elements of appropriate subdifferentials.

Sets (30) - (32) are called the limiting (Mordukhovich) subdifferential [38,48], limiting outer subdifferential [25], and uniform limiting subdifferential of $f$ at $\bar{x}$ respectively. See [45] for an infinitedimensional generalization of (31) and [38, Definition 1.100] for a closely related definition of the "right-sided" subdifferential.

Proposition 12 (i). $\overline{|\partial f|}(\bar{x})=\inf \left\{\left\|x^{*}\right\|: x^{*} \in \bar{\partial} f(\bar{x})\right\}$.

(ii). $\overline{|\partial f|}>(\bar{x})=\inf \left\{\left\|x^{*}\right\|: x^{*} \in \bar{\partial}^{>} f(\bar{x})\right\}$.

(iii). $|\overline{|\partial f|}|^{\diamond}(\bar{x})=\inf \left\{\left\|x^{*}\right\|: x^{*} \in \bar{\partial}^{\diamond} f(\bar{x})\right\}$.

(iv). $\overline{|\partial f|}(\bar{x})>0$ if and only if $0 \notin \bar{\partial} f(\bar{x})$.

(v). $|\overline{|\partial f|}|^{>}(\bar{x})>0$ if and only if $0 \notin \bar{\partial}^{>} f(\bar{x})$.

(vi). $\left.\overline{|\partial f|}\right|^{\diamond}(\bar{x})>0$ if and only if $0 \notin \bar{\partial}^{\diamond} f(\bar{x})$.

Proof Assertions (i) - (iii) follow from definitions (22) - (24), (28) - (32), while (iv) - (vi) are

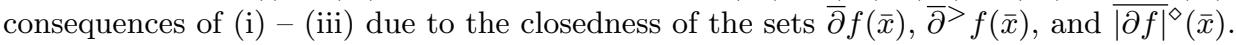

The next proposition provides an important example of a regular set of subgradients and a simplified representation of the internal subdifferential slope (25) strengthening Proposition 9.

Proposition 13 (i). Every bounded subset of $\partial f(\bar{x})$ is a regular set of subgradients of $f$ at $\bar{x}$.

(ii). $|\partial f|^{0}(\bar{x})=\sup \left\{r \geq 0: r \mathbb{B}^{*} \in \partial f(\bar{x})\right\}$.

(iii). $|\partial f|^{0}(\bar{x})>0$ if and only if $0 \in \operatorname{int} \partial f(\bar{x})$.

Proof (i) Let $G$ be a bounded subset of $\partial f(\bar{x})$ which is not a regular set of subgradients of $f$ at $\bar{x}$. By definition, there exists an $\varepsilon>0$ and sequences $x_{k} \rightarrow \bar{x}$ and $\left\{x_{k}^{*}\right\} \subset G$ such that

$$
f\left(x_{k}\right)-f(\bar{x})-\left\langle x_{k}^{*}, x_{k}-\bar{x}\right\rangle+2 \varepsilon\left\|x_{k}-\bar{x}\right\|<0 .
$$

Without loss of generality $x_{k}^{*}$ converges to some $x^{*}$ which must belong to $\partial f(\bar{x})$ since the latter set is closed. On the other hand, $\left\|x_{k}^{*}-x^{*}\right\|<\varepsilon$ for all sufficiently large $k$, and it follows from the last inequality that

$$
f\left(x_{k}\right)-f(\bar{x})-\left\langle x^{*}, x_{k}-\bar{x}\right\rangle+\varepsilon\left\|x_{k}-\bar{x}\right\|<0,
$$

and consequently $x^{*} \notin \partial f(\bar{x})$. This contradiction completes the proof.

(ii) follows from (i) and definition (25).

(iii) follows from (ii). 
Thanks to Proposition 12 (iv)-(vi) and Proposition 13 (iii) we can formulate the finite dimensional versions of criteria $\mathrm{C} 7, \mathrm{C} 4, \mathrm{C} 10$, and $\mathrm{NC} 2$.

C9 $0 \notin \bar{\partial} f(\bar{x})$.

C10 $0 \notin \bar{\partial}^{>} f(\bar{x})$.

C11 $0 \in \operatorname{int} \partial f(\bar{x})$.

NC3 $0 \notin \bar{\partial}^{\diamond} f(\bar{x})$.

Criterion C11 is in general independent of C10 and C9. The "combined" sufficient criteria

$$
0 \notin \bar{\partial} f(\bar{x}) \backslash \operatorname{int} \partial f(\bar{x}), \quad 0 \notin \bar{\partial}^{>} f(\bar{x}) \backslash \operatorname{int} \partial f(\bar{x})
$$

can be of interest. The first one can be rewritten as

C12 $0 \notin \operatorname{bd} \partial f(\bar{x})$,

if $f$ is lower regular [38, Definition 1.91] at $\bar{x}$, that is, if $\bar{\partial} f(\bar{x})=\partial f(\bar{x})$.

Criterion C12 was used in [18, Corollary 3.4] and [19, Theorem 4.2].

The implication $\mathrm{C} 11 \Rightarrow \mathrm{C} 10$ (and consequently $\mathrm{C} 10 \Rightarrow \mathrm{C} 4$ ) holds true for semismooth functions. We recall from [37] that a function $f$ on a finite dimensional space $X$ is semismooth at $\bar{x}$ if it is Lipschitz continuous around $\bar{x}$ and for any sequences $z_{i} \rightarrow z \in X, t_{i} \downarrow 0, x_{i}^{*} \in \partial_{C} f\left(\bar{x}+t_{i} z_{i}\right)$ (Clarke subdifferential of $f$ at $\left.\bar{x}+t_{i} z_{i}\right)$ one has $\left\langle x_{i}^{*}, z\right\rangle \rightarrow f^{\prime}(\bar{x} ; z)$ (the derivative of $f$ at $\bar{x}$ in the direction $z$ ). As proved in [28], each subanalytic function, Lipschitz around $\bar{x}$, is semismooth at $\bar{x}$.

Proposition 14 Let $f: X \rightarrow \mathbb{R}$ be semismooth at $\bar{x}$. Then

(i). int $\partial f(\bar{x}) \cap \bar{\partial}^{>} f(\bar{x})=\emptyset$;

(ii). if $0 \in \operatorname{int} \partial f(\bar{x})$ then $0 \notin \bar{\partial}^{>} f(\bar{x})$;

(iii). $|\partial f|^{0}(\bar{x}) \leq \overline{|\partial f|}>(\bar{x})$.

Proof Let $x^{*} \in \bar{\partial}^{>} f(\bar{x})$. By definition (31), there exist sequences $x_{i} \rightarrow \bar{x}$ with $f\left(x_{i}\right) \downarrow f(\bar{x})$ and $x_{i}^{*} \rightarrow x^{*}$ with $x_{i}^{*} \in \partial f\left(x_{i}\right)$. Denote $t_{i}=\left\|x_{i}-\bar{x}\right\|, z_{i}=\left(x_{i}-\bar{x}\right) / t_{i}$. Then $t_{i} \downarrow 0$ and without loss of generality we can assume that $z_{i}$ converges to some $z \in X,\|z\|=1$. By the semismoothness of $f,\left\langle x_{i}^{*}, z\right\rangle \rightarrow f^{\prime}(\bar{x} ; z)$, and consequently $f^{\prime}(\bar{x} ; z)=\left\langle x^{*}, z\right\rangle$. On the other hand, due to the Lipschitz continuity of $f, f^{\prime}(\bar{x} ; z) \geq\left\langle v^{*}, z\right\rangle$ for any $v^{*} \in \partial f(\bar{x})$. This means that $x^{*} \notin \operatorname{int} \partial f(\bar{x})$. The proof of the first assertion is completed. The other two assertions are obvious corollaries of the first one.

The relationships among the error bound criteria for a lower semicontinuous function on a finite dimensional space are illustrated in Fig. 5.

The efficiency of criterion C10 depends on the ability to compute a tight upper estimate of $\bar{\partial}^{>} f(\bar{x})$. This is possible, for instance, in the following important situation.

Let $C \subset \mathbb{R}^{m}$ be closed and $\varphi: \mathbb{R}^{m} \rightarrow \mathbb{R}_{+}$be locally Lipschitz continuous and satisfy the condition

$$
\varphi(y)=0 \quad \Leftrightarrow \quad y \in C .
$$

Assume that $F: \mathbb{R}^{n} \rightarrow \mathbb{R}^{m}$ is continuously differentiable and define the composition $f: \mathbb{R}^{n} \rightarrow \mathbb{R}_{+}$ by $f(x)=\varphi(F(x))$.

Theorem 4 Let $\bar{x} \in \mathbb{R}^{n}$ and $\bar{y}=F(\bar{x}) \in C$. Then

$$
\bar{\partial}^{>} f(\bar{x}) \subset(\nabla F(\bar{x}))^{*} \operatorname{Limsup}_{x \rightarrow \bar{x}, F(x) \notin C} \bar{\partial} \varphi(F(x)) .
$$

Inclusion (33) becomes equality, provided one of the following conditions holds true:

(a) $\nabla F(\bar{x})$ is surjective;

(b) $\varphi$ is lower regular on a neighborhood of $\bar{y}$, that is, $\bar{\partial} \varphi(y)=\partial \varphi(y)$ for all $y$ near $\bar{y}$.

In case (b) one has

$$
\bar{\partial}^{>} f(\bar{x})=(\nabla F(\bar{x}))^{*} \underset{x \rightarrow \bar{x}, F(x) \notin C}{\operatorname{Limsup}} \partial \varphi(F(x)) .
$$




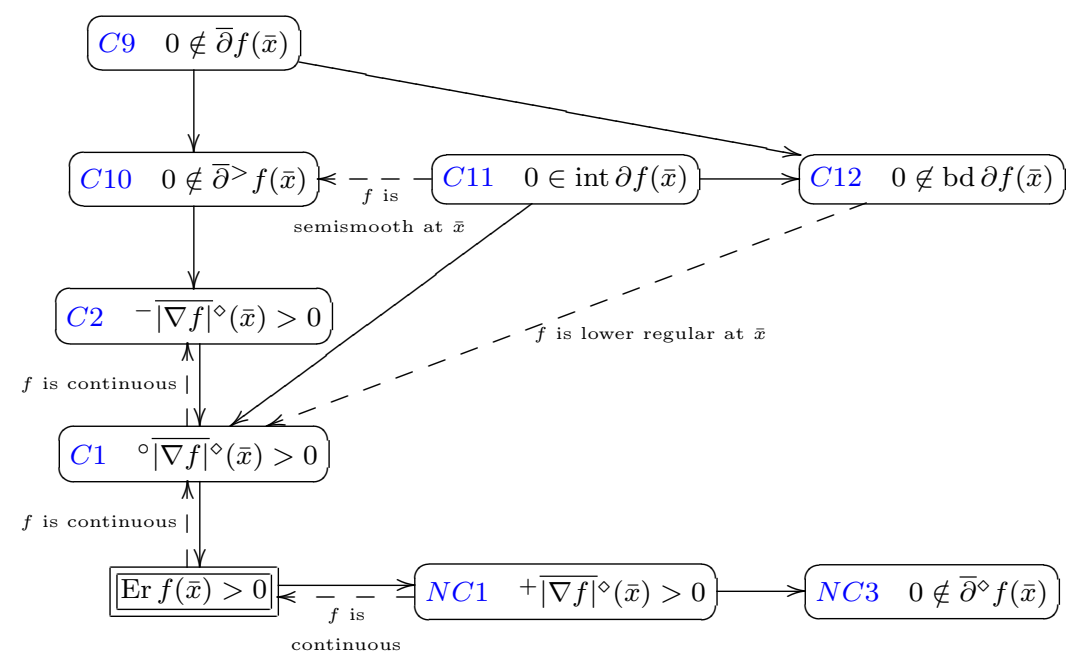

Fig. 5 Finite dimensional case

Proof By definition (31),

$$
\bar{\partial}^{>} f(\bar{x})=\operatorname{Limsup}_{x \rightarrow \bar{x}, F(x) \notin C} \partial f(x) .
$$

Since $F$ is Lipschitz near $\bar{x}$ and $\varphi$ is Lipschitz near $\bar{y}$, it holds by virtue of [48, Theorem 10.49] that

$$
\partial f(x) \subset(\nabla F(x))^{*} \bar{\partial} \varphi(F(x)) .
$$

Moreover, due to the uniform boundedness of $\bar{\partial} \varphi$,

$$
\bar{\partial}^{>} f(\bar{x}) \subset \operatorname{Limsup}_{x \rightarrow \bar{x}, F(x) \notin C}(\nabla F(x))^{*} \bar{\partial} \varphi(F(x))=(\nabla F(\bar{x}))^{*} \operatorname{Limsup}_{x \rightarrow \bar{x}, F(x) \notin C} \bar{\partial} \varphi(F(x)) .
$$

To prove that (33) becomes equality under (a), we recall from [48, Theorem 10.49] the estimate

$$
\partial f(x) \supset(\nabla F(x))^{*} \partial \varphi(F(x)) .
$$

This implies, by the uniform boundedness of $\partial \varphi$, that

$$
\bar{\partial}^{>} f(\bar{x}) \supset \operatorname{Limsup}_{x \rightarrow \bar{x}, F(x) \notin C}(\nabla F(x))^{*} \partial \varphi(F(x))=(\nabla F(\bar{x}))^{*} \underset{x \rightarrow \bar{x}, F(x) \notin C}{\operatorname{Limsup}} \partial \varphi(F(x)) .
$$

We show that the sets in the right-hand sides of (33) and (34) are the same. To this end, consider a vector $\xi \in \operatorname{Limsup}_{x \rightarrow \bar{x}, F(x) \notin C} \bar{\partial} \varphi(F(x))$ given by

$$
\xi=\lim _{i \rightarrow \infty} \xi_{i} \quad \text { with } \xi_{i} \in \bar{\partial} \varphi\left(F\left(x_{i}\right)\right), x_{i} \rightarrow \bar{x}, F\left(x_{i}\right) \notin C .
$$

By the definition of the limiting subdifferential and by the closedness of $C$, for each $i$ there are sequences $z_{i j} \rightarrow F\left(x_{i}\right), \varrho_{i j} \rightarrow \xi_{i}$ such that $z_{i j} \notin C$ and $\varrho_{i j} \in \partial \varphi\left(z_{i j}\right)$. Due to the surjectivity of $\nabla F(\bar{x})$, for sufficiently large $i, j$ one has that $z_{i j}=F\left(x_{i j}\right)$ for some $x_{i j} \rightarrow x_{i}$. By considering "diagonal" sequences $\tilde{x}_{i}=x_{i i}, \eta_{i}=\varrho_{i i}$ we now easily conclude that $\xi \in \underset{x \rightarrow \bar{x}, F(x) \notin C}{\operatorname{Limsup}} \partial \varphi(F(x))$ and we are done.

Under (b) the result follows immediately from inclusions (33) and (34).

In concrete situations we may sometimes also use the evident estimates

$$
\bar{\partial}^{>} f(\bar{x}) \subset(\nabla F(\bar{x}))^{*} \operatorname{Limsup}_{y \in \operatorname{Im} F \backslash C} \partial \varphi(y) \subset(\nabla F(\bar{x}))^{*} \bar{\partial}^{>} \varphi(\bar{y}) .
$$

In finite dimensions Proposition 4 improves the sufficient condition given in [45, Corollary 5.4]. Such an improvement can be important as shown in the next example. 
Example 12 Let $C=\mathbb{R}_{+}^{2}$ and a mapping $F: \mathbb{R} \rightarrow \mathbb{R}^{2}$ be defined as $F(y)=(-y, y)^{T}$. Consider the composition $f=d_{C} \circ F$, where $d_{C}$ is the Euclidean distance to the set $C$. By Theorem 4 ,

$$
\bar{\partial}^{>} f(0)=(-1,1) \cdot\left\{(0,-1)^{T},(-1,0)^{T}\right\}
$$

and consequently $\operatorname{Er} f(0) \geq \overline{|\partial f|}>(0)>0$.

On the other hand, $\bar{\partial}^{>} d_{C}(0)=\left\{x \in \mathbb{R}_{-}^{2}:\|x\|=1\right\}$, and we observe that $0 \in(-1,1) \cdot \bar{\partial}^{>} d_{C}(0)$. Consequently, the estimate in [45, Corollary 5.4] does not enable us to detect that $\operatorname{Er} f(0)>0$.

\section{Convex Case}

In this section $X$ is a general Banach space and $f$ is convex lower semicontinuous.

In the convex case many constants considered in the preceding sections coincide.

Theorem 5 (i). $\overline{|\nabla f|}(\bar{x})=\overline{|\partial f|}(\bar{x})=|\nabla f|(\bar{x})=|\partial f|(\bar{x})$.

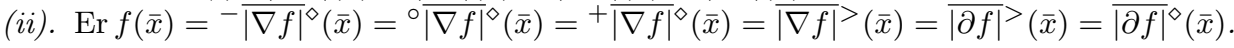

(iii). $|\partial f|(\bar{x})>0$ if and only if $0 \notin \partial f(\bar{x})$.

Proof (i) Thanks to Propositions 3 and 5 (i), it holds

$$
|\nabla f|(\bar{x}) \leq|\partial f|(\bar{x}) \quad \text { and } \quad \overline{|\nabla f|}(\bar{x}) \leq \overline{|\partial f|}(\bar{x}) \leq|\partial f|(\bar{x}) .
$$

We are going to show that

$$
|\partial f|(\bar{x}) \leq|\nabla f|(\bar{x}) \leq \overline{|\nabla f|}(\bar{x}) .
$$

To prove the first inequality we only need to show that inequality $|\nabla f|(\bar{x})<\gamma$ implies the existence of an $x^{*} \in \partial f(\bar{x})$ with $\left\|x^{*}\right\| \leq \gamma$. If $|\nabla f|(\bar{x})<\gamma$ then by (9), $\bar{x}$ is a point of local minimum of the function $x \mapsto g(x)=f(x)+\gamma\|x-\bar{x}\|$, and consequently $0 \in \partial g(\bar{x})$. Observing that $g$ is a sum of two convex functions one of which is continuous and applying the convex sum rule, we conclude that there exists an $x^{*} \in \partial f(\bar{x})$ with $\left\|x^{*}\right\| \leq \gamma$.

The second inequality holds trivially if $\overline{|\nabla f|}(\bar{x})=\infty$. Let $\overline{|\nabla f|}(\bar{x})<\gamma<\infty$. Then by definition (17), there is a sequence $x_{k} \rightarrow \bar{x}$ such that $f\left(x_{k}\right) \rightarrow f(\bar{x})$ and

$$
f(x)-f\left(x_{k}\right)+\gamma\left\|x-x_{k}\right\| \geq 0
$$

for all $x$ near $x_{k}$. Due to convexity of $f$ the last inequality holds true for all $x \in X$. Passing to the limit as $k \rightarrow \infty$ and recalling the definition (9) of the slope, we conclude that $|\nabla f|(\bar{x}) \leq \gamma$, and consequently $|\nabla f|(\bar{x}) \leq \overline{|\nabla f|}(\bar{x})$.

(ii) For any $x \in X$ with $f(x)>f(\bar{x})$ and any $\alpha>0$ it holds

$$
\begin{aligned}
\sup _{0<\|u-x\|<d(x,[f \leq f(\bar{x})])} \frac{(f(x)-f(u))_{+}}{\|u-x\|} & =\sup _{0<\|u-x\|<\alpha} \frac{(f(x)-f(u))_{+}}{\|u-x\|} \\
& =\sup _{u \neq x, f(u) \geq f(\bar{x})} \frac{(f(x)-f(u))_{+}}{\|u-x\|}=\limsup _{u \rightarrow x} \frac{(f(x)-f(u))_{+}}{\|u-x\|} .
\end{aligned}
$$

Equalities $-\overline{\mid \nabla f}^{\diamond}(\bar{x})={ }^{\circ} \overline{|\nabla f|^{\diamond}}(\bar{x})=\left.{ }^{+} \overline{|\nabla f|}\right|^{\diamond}(\bar{x})=\overline{|\nabla f|}^{>}(\bar{x})$ follow now from definitions (6) - (8) and (18).

By virtue of (i), we have $|\nabla f|(x)=|\partial f|(x)$ for all $x \in X$. Equality $\overline{|\nabla f|}^{>}(\bar{x})=\overline{\mid \partial f}^{>}(\bar{x})$ follows from comparing definitions (18) and (24).

It is not difficult to see that $\partial_{\varepsilon, \alpha}^{\diamond} f(\bar{x})$ does not depend on $\alpha$ and equals $\partial f(\bar{x})+\varepsilon \mathbb{B}^{*}$. This observation yields equality $\overline{|\partial f|^{\diamond}}(\bar{x})=\overline{|\partial f|}>(\bar{x})$.

Equality $\operatorname{Er} f(\bar{x})=-\overline{|\nabla f|^{\diamond}}(\bar{x})$ is a consequence of Theorem 2.

(iii) is a consequence of (i) due to the closedness of the set $\partial f(\bar{x})$.

Equality $|\nabla f|(\bar{x})=|\partial f|(\bar{x})$ in Theorem 5 (i) is well known - see [2, Remark 2.1], [3, Proposition 2.5], [4, Proposition 3.1], [13, Proposition 5.2].

Due to Theorem 5, a number of sufficient criteria formulated in the preceding sections reduce in the convex case to two conditions: criterion $\mathrm{C} 4$ and the following one: 
C13 $0 \notin \partial f(\bar{x})$.

Criterion C4 is also necessary and can be strictly weaker than C13, see the function in Example 6. The next assertion follows from Proposition 7 and definition (25).

Proposition $15(i) . \partial f(\bar{x})$ is a regular set of subgradients of $f$ at $\bar{x}$.

(ii). $|\partial f|^{0}(\bar{x})=\sup \left\{r \geq 0: r \mathbb{B}^{*} \in \partial f(\bar{x})\right\}$.

(iii). $|\partial f|^{0}(\bar{x})>0$ if and only if $0 \in \operatorname{int} \partial f(\bar{x})$.

Thanks to Proposition 15, the finite dimensional sufficient criterion C11 is applicable to convex functions in infinite dimensions.

Conditions C11 and C13 are mutually exclusive and can be replaced by a single criterion C12, which is still in general stronger than $\mathrm{C} 4$.

The relationships among the error bound criteria for a lower semicontinuous convex function on a Banach space are illustrated in Fig. 6.

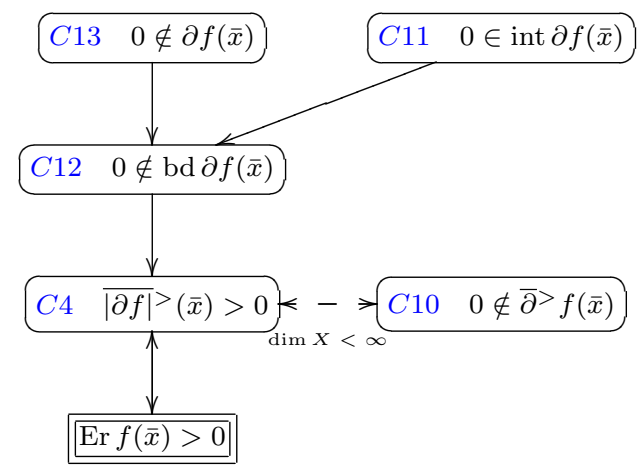

Fig. 6 Convex case

Criterion C4 was used in [25, Theorem 2.1(c)], [43, Corollary 2(ii)], [45, Theorem 4.12], [50, Theorem 3.1]. Criterion C12 was used in [18, Corollary 3.4], [19, Theorem 4.2]. The equality Er $f(\bar{x})=\overline{|\partial f|}^{>}(\bar{x})$ seems to be well known as well.

Being stronger than $\mathrm{C} 4$, criterion $\mathrm{C} 12$ characterizes a stronger property than just the existence of a local error bound for $f$ at $\bar{x}$, namely, it guaranties the local error bound property for a family of functions being small perturbations of $f$, see [41].

Acknowledgements The authors wish to thank Abderrahim Jourani, Bernd Kummer, and David Yost for fruitful discussions while preparing the paper and the anonymous referee for very careful reading of the paper and many valuable comments and suggestions, which helped us improve the presentation.

\section{References}

1. Azé, D.: A survey on error bounds for lower semicontinuous functions. In: Proceedings of 2003 MODESMAI Conference, ESAIM Proc., vol. 13, pp. 1-17. EDP Sci., Les Ulis (2003)

2. Azé, D.: A unified theory for metric regularity of multifunctions. J. Convex Anal. 13(2), 225-252 (2006)

3. Azé, D., Corvellec, J.N.: On the sensitivity analysis of Hoffman constants for systems of linear inequalities. SIAM J. Optim. 12(4), 913-927 (2002)

4. Azé, D., Corvellec, J.N.: Characterizations of error bounds for lower semicontinuous functions on metric spaces. ESAIM Control Optim. Calc. Var. 10(3), 409-425 (2004)

5. Bosch, P., Jourani, A., Henrion, R.: Sufficient conditions for error bounds and applications. Appl. Math. Optim. 50(2), 161-181 (2004)

6. Burke, J.V.: Calmness and exact penalization. SIAM J. Control Optim. 29(2), $493-497$ (1991)

7. Burke, J.V., Deng, S.: Weak sharp minima revisited. I. Basic theory. Control Cybernet. 31(3), 439-469 (2002). Well-Posedness in Optimization and Related Topics (Warsaw, 2001)

8. Burke, J.V., Deng, S.: Weak sharp minima revisited. II. Application to linear regularity and error bounds. Math. Program., Ser. B 104(2-3), 235-261 (2005)

9. Burke, J.V., Ferris, M.C.: Weak sharp minima in mathematical programming. SIAM J. Control Optim. 31(5), 1340-1359 (1993) 
10. Clarke, F.H.: A new approach to Lagrange multipliers. Math. Oper. Res. 1(2), 165-174 (1976)

11. Clarke, F.H.: Optimization and Nonsmooth Analysis. Canadian Mathematical Society Series of Monographs and Advanced Texts. John Wiley \& Sons Inc., New York (1983). A Wiley-Interscience Publication

12. Cornejo, O., Jourani, A., Zălinescu, C.: Conditioning and upper-Lipschitz inverse subdifferentials in nonsmooth optimization problems. J. Optim. Theory Appl. 95(1), 127-148 (1997)

13. Corvellec, J.N., Motreanu, V.V.: Nonlinear error bounds for lower semicontinuous functions on metric spaces. Math. Program., Ser. A 114(2), 291-319 (2008)

14. De Giorgi, E., Marino, A., Tosques, M.: Problems of evolution in metric spaces and maximal decreasing curve. Atti Accad. Naz. Lincei Rend. Cl. Sci. Fis. Mat. Natur. (8) 68(3), 180-187 (1980)

15. Deng, S.: Global error bounds for convex inequality systems in Banach spaces. SIAM J. Control Optim. 36(4), 1240-1249 (1998)

16. Dontchev, A.L., Rockafellar, R.T.: Regularity and conditioning of solution mappings in variational analysis. Set-Valued Anal. 12(1-2), 79-109 (2004)

17. Fabian, M., Habala, P., Hájek, P., Montesinos Santalucía, V., Pelant, J., Zizler, V.: Functional Analysis and Infinite-Dimensional Geometry. CMS Books in Mathematics/Ouvrages de Mathématiques de la SMC, 8. Springer-Verlag, New York (2001)

18. Henrion, R., Jourani, A.: Subdifferential conditions for calmness of convex constraints. SIAM J. Optim. $\mathbf{1 3}(2), 520-534(2002)$

19. Henrion, R., Outrata, J.V.: A subdifferential condition for calmness of multifunctions. J. Math. Anal. Appl. 258(1), 110-130 (2001)

20. Henrion, R., Outrata, J.V.: Calmness of constraint systems with applications. Math. Program. 104(2-3, Ser. B), 437-464 (2005)

21. Hoffman, A.J.: On approximate solutions of systems of linear inequalities. J. Research Nat. Bur. Standards 49, 263-265 (1952)

22. Ioffe, A.D.: Necessary and sufficient conditions for a local minimum. I. A reduction theorem and first order conditions. SIAM J. Control Optim. 17(2), 245-250 (1979)

23. Ioffe, A.D.: Regular points of Lipschitz functions. Trans. Amer. Math. Soc. 251, 61-69 (1979)

24. Ioffe, A.D.: Metric regularity and subdifferential calculus. Russian Math. Surveys 55, 501-558 (2000)

25. Ioffe, A.D., Outrata, J.V.: On metric and calmness qualification conditions in subdifferential calculus Set-Valued Anal. 16(2-3), 199-227 (2008)

26. Jourani, A.: Hoffman's error bound, local controllability, and sensitivity analysis. SIAM J. Control Optim. 38(3), 947-970 (2000)

27. Jourani, A.: Weak regularity of functions and sets in Asplund spaces. Nonlinear Anal. 65(3), 660-676 (2006)

28. Jourani, A.: Radiality and semismoothness. Control Cybernet. 36(3), 669-680 (2007)

29. Klatte, D., Kummer, B.: Nonsmooth Equations in Optimization, Nonconvex Optimization and its Applications, vol. 60. Kluwer Academic Publishers, Dordrecht (2002). Regularity, calculus, methods and applications

30. Klatte, D., Kummer, B.: Stability of inclusions: characterizations via suitable Lipschitz functions and algorithms. Optimization 55(5-6), 627-660 (2006)

31. Kruger, A.Y.: Generalized differentials of nonsmooth functions. Deposited in VINITI no. 1332-81. Minsk (1981). In Russian

32. Kruger, A.Y.: $\varepsilon$-semidifferentials and $\varepsilon$-normal elements. Deposited in VINITI no. 1331-81. Minsk (1981). In Russian

33. Kruger, A.Y.: Strict $(\varepsilon, \delta)$-subdifferentials and extremality conditions. Optimization 51(3), 539-554 (2002)

34. Kruger, A.Y.: On Fréchet subdifferentials. J. Math. Sci. (N. Y.) 116(3), 3325-3358 (2003). Optimization and Related Topics, 3

35. Lewis, A.S., Pang, J.S.: Error bounds for convex inequality systems. In: Generalized Convexity, Generalized Monotonicity: Recent Results (Luminy, 1996), Nonconvex Optim. Appl., vol. 27, pp. 75-110. Kluwer Acad. Publ., Dordrecht (1998)

36. Łojasiewicz, S.: Sur le problème de la division. Studia Math. 18, 87-136 (1959)

37. Mifflin, R.: Semismooth and semiconvex functions in constrained optimization. SIAM J. Control Optimization 15(6), 959-972 (1977)

38. Mordukhovich, B.S.: Variational Analysis and Generalized Differentiation. I: Basic Theory, Grundlehren der Mathematischen Wissenschaften [Fundamental Principles of Mathematical Sciences], vol. 330. Springer-Verlag, Berlin (2006)

39. Ng, K.F., Yang, W.H.: Regularities and their relations to error bounds. Math. Program., Ser. A 99, 521-538 (2004)

40. Ng, K.F., Zheng, X.Y.: Error bounds for lower semicontinuous functions in normed spaces. SIAM J. Optim. 12(1), 1-17 (2001)

41. Ngai, H.V., Kruger, A.Y., Théra, M.: Stability of error bounds for semi-infinite convex constraint systems. SIAM J. Optim. 20 (2010)

42. Ngai, H.V., Théra, M.: Error bounds in metric spaces and application to the perturbation stability of metric regularity. SIAM J. Optim. 19(1), 1-20 (2008)

43. Ngai, H.V., Théra, M.: Error bounds for systems of lower semicontinuous functions in Asplund spaces. Math. Program., Ser. B 116(1-2), 397-427 (2009)

44. Pang, J.S.: Error bounds in mathematical programming. Math. Programming, Ser. B 79(1-3), 299-332 (1997). Lectures on Mathematical Programming (ISMP97) (Lausanne, 1997)

45. Penot, J.P.: Error bounds, calmness and their applications in nonsmooth analysis. Contemporary Mathematics (2010). To be published 
46. Polyak, B.T.: Introduction to Optimization. Translations Series in Mathematics and Engineering. Optimization Software Inc. Publications Division, New York (1987). Translated from Russian

47. Rockafellar, R.T.: Directionally Lipschitzian functions and subdifferential calculus. Proc. London Math. Soc. (3) 39(2), 331-355 (1979)

48. Rockafellar, R.T., Wets, R.J.B.: Variational Analysis, Grundlehren der Mathematischen Wissenschaften [Fundamental Principles of Mathematical Sciences], vol. 317. Springer-Verlag, Berlin (1998)

49. Studniarski, M., Ward, D.E.: Weak sharp minima: characterizations and sufficient conditions. SIAM J. Control Optim. 38(1), 219-236 (1999)

50. Wu, Z., Ye, J.J.: Sufficient conditions for error bounds. SIAM J. Optim. 12(2), 421-435 (2001/02)

51. Wu, Z., Ye, J.J.: On error bounds for lower semicontinuous functions. Math. Program., Ser. A 92(2), 301-314 (2002)

52. Ye, J.J., Ye, X.Y.: Necessary optimality conditions for optimization problems with variational inequality constraints. Math. Oper. Res. 22(4), 977-997 (1997) 